\title{
Simpler Efficient Group Signatures from Lattices
}

\author{
Phong Q. Nguyen ${ }^{1,2}$, Jiang Zhang ${ }^{3}$, and Zhenfeng Zhang ${ }^{3(凶)}$ \\ 1 INRIA, Paris, France \\ pnguyen@di.ens.fr, \\ http://www.di.ens.fr/ pnguyen \\ 2 Institute for Advanced Study, Tsinghua University, Beijing, China \\ 3 Trusted Computing and Information Assurance Laboratory, \\ State Key Laboratory of Computer Science, Institute of Software, \\ Chinese Academy of Sciences, Beijing, China \\ jiangzhang09@gmail.com, zfzhang@tca.iscas.ac.cn
}

\begin{abstract}
A group signature allows a group member to anonymously sign messages on behalf of the group. In the past few years, new group signatures based on lattice problems have appeared: the most efficient lattice-based constructions are due to Laguillaumie et al. (Asiacrypt '13) and Langlois et al. (PKC '14). Both have at least $O\left(n^{2} \log ^{2} n \log N\right)$ bit group public key and $O\left(n \log ^{3} n \log N\right)$-bit signature, where $n$ is the security parameter and $N$ is the maximum number of group members. In this paper, we present a simpler lattice-based group signature, which is more efficient by a $O(\log N)$ factor in both the group public key and the signature size. We achieve this by using a new non-interactive zeroknowledge (NIZK) proof corresponding to a simple identity-encoding function. The security of our group signature can be reduced to the hardness of SIS and LWE in the random oracle model.
\end{abstract}

\section{Introduction}

In a group signature, each group member has a private key that is certified with its identity by the group manager. By using its private key, each group member is able to sign messages on behalf of the group without compromising its identity to the signature verifier. Group signatures provide users a nice tradeoff between authenticity and anonymity (i.e., given a signature, the verifier is assured that someone in the group signed a message, but cannot determine which member of the group signed). However, such a functionality allows malicious group members to damage the whole group without being detected, e.g. signing some unauthorized/illegal messages. To avoid this, the group manager usually has a secret key which can be used to break anonymity.

Several real-life applications require properties of group signatures. For example, in trusted computing, a trusted platform module (TPM) usually has to attest

The work is supported in part by China's 973 program (No. 2013CB338003, 2013CB834205) and the National Natural Science Foundation of China (No. 61133013, 61170278, 91118006). 
certain statements w.r.t. the current configurations of the host device to a remote party (i.e. the verifier) via a signature on corresponding messages. After the attestation, the verifier is assured that some remote device that contains a TPM authorized the messages. For user privacy, the signature is often required not to reveal the identity of the TPM. In fact, a variant of group signatures (namely, direct anonymous attestation (DAA) $[26,33]$ ) has been implemented in TPM 1.2 [40] and TPM 2.0 [41] by the Trusted Computing Group. Another promising application is vehicle safety communications [42], where group signatures can protect the privacy of users so that a broadcast message does not reveal the current location/speed of the vehicle. Besides, other applications of group signatures are found in anonymous communications, e-commerce systems etc.

Since their introduction by Chaum and van Heyst [32], group signatures have attracted much attention from the research community. Bellare, Micciancio and Warinschi (BMW) [11] formalized the security of group signatures for static groups (where the group members are fixed in the system setup phase) in two main notions, i.e., full anonymity and full traceability. Informally, full anonymity requires that an adversary without the group manager secret key should not be able to determine the signer's identity from a signature, even if it can access an open oracle that returns the identity of any other (valid) signature. And full traceability implies that no collusion of group members can create a valid signature which cannot be traced back to one of them (by the group manager using the group manager secret key). Bellare et al. [11] also gave a theoretical construction based on the existence of trapdoor permutations. In a weak variant of the BMW model where the adversary against anonymity is not given access to the open oracle (i.e., CPA-anonymity), Boneh et al. [16] constructed a short group signature scheme based on the Strong Diffie-Hellman (SDH) [15] and Decision Linear (DLIN) [16] assumptions in the random oracle model [13]. Besides, many papers focused on designing various group signatures based on different assumptions $[1,9,20,21,28,38,39,45,46]$.

In recent years, lattice cryptography has attracted significant interest, due to several potential benefits: asymptotic efficiency, worst-case hardness assumptions, and security against quantum computers. A natural goal is to find latticebased counterparts of all classical cryptographic schemes. In 2010, Gordon et al. [37] made the first step in constructing secure group signatures from lattices. They elegantly combined several powerful lattice-based tools $[36,54,57]$ to build a group signature scheme where the sizes of both the group public key and signature was linear in the maximum number $N$ of group members. Later, Camenisch el al. [29] proposed a variant of [37] with improvements both in efficiency (i.e., shorter group public key) and security (i.e., stronger adversary against anonymity), but the signature size of their scheme was still linear in $N$. Recently, two papers $[43,44]$ have significantly decreased the signature size. By first representing the identity of group members as a bit-string [19], and then applying the "encrypt-and-prove" paradigm of [11,37], Laguillaumie et al. [43] constructed an efficient lattice-based group signature where both the sizes of the group public key and the signature are proportional to $\log N$ (i.e., with 
bit-length slightly greater than $O\left(n^{2} \log ^{2} n \log N\right)$ and $O\left(n \log ^{3} n \log N\right)$, respectively). Using similar identity representations together with the non-interactive zero-knowledge (NIZK) proof in [47], Langlois et al. [44] proposed a nice scheme without encryption, which achieves almost the same asymptotical efficiency as that of [43], and provides an additional property called verifier-local revocation [18]. Another interesting group signature is due to Benhamouda et al. [14], for which privacy holds under a lattice-based assumption but the security is discrete-logarithm-based, i.e. it is not a pure lattice-based group signature.

A current and independent work of Ling, Nguyen and Wang [48] also try to design an efficient lattice-based group signature scheme. Specifically, by first constructing a nice Stern-type [58] NIZK protocol, they propose a scheme which excels previous ones in $[43,44]$ by a constant factor in terms of efficiency, i.e., all the sizes are still proportional to $\log N$. Besides, they also show how to transform their basic scheme into the setting of ideal lattices, which can save a factor of $n$ in the size of group public key.

\subsection{Our Results}

In this paper, we present a new lattice-based group signature. Compared to the best previous lattice-based schemes $[37,43,44]$, it is both simpler and more efficient, saving a $O(\log N)$ factor in both sizes of the group public key and the signature. As in [43], we first present a simple CPA-anonymous scheme, which can be easily extended to support CCA-anonymity (please refer to the full version). The security of both our schemes is provably based on the hardness of the Small Integer Solutions (SIS) and Learning with Errors (LWE) problems in the random oracle model, which are both as hard as several worst-case lattice problems, such as $\mathrm{SIVP}_{\gamma}$ for some polynomial factor $\gamma=\operatorname{poly}(n)$.

In Table 1, we give a rough comparison with related lattice-based group signatures in terms of the size of the group public-key, the group user secret key and the signature. There, $n$ denotes the security parameter, and $N$ is the maximum number of group users. The other two parameters $m$ and $q$ are both polynomial in $n$ (and $N$ ), and are usually determined by the underlying lattices used by those schemes. The integer $t$ used in $[43,44]$ and our scheme is a repetition parameter for obtaining NIZKs with negligible soundness error. For a security parameter $n$, one can set $t=\omega(\log n)$ and $m=O(n \log n)$. The choice of $q$ might be slightly different in those schemes either for security or for functionality. For example, $q$ is explicitly required to be larger than $N$ in our scheme. We note that this requirement might also be satisfied in the previous three schemes for most applications. Besides, even if $N<q$ does not hold in previous schemes, the sizes of the group public-key and the signature in our scheme are still asymptotically shorter (since both $N$ and $q$ are polynomials in $n$, and $\log N=O(\log q)$ holds).

Since the schemes in $[37,43]$ and ours follow a general "encrypt-and-prove" paradigm in [11], we also give a comparison of computational costs between the schemes in $[37,43]$ and ours at a very high level, i.e., in terms of the number of the underlying encryptions and basic NIZK proofs, in Table 2. We note that such a comparison is less interesting to the scheme in [44], since it departs from the 
Table 1. Rough Comparison of Overheads

\begin{tabular}{|c|c|c|c|c|}
\hline Schemes & Group public-key & User secret-key & Signature & Security \\
\hline GKV10 [37] & $O(n m N \log q)$ & $O(n m \log q)$ & $O(n m N \log q)$ & CPA-anonymity \\
\hline LLLS13 [43] & $O(n m \log N \log q)$ & $O(n m \log q)$ & $O(t m \log N \log q)$ & CCA-anonymity \\
\hline LLNW14 [44] & $O(n m \log N \log q)$ & $O(m \log N \log q)$ & $O(t m \log N \log q \log \beta)^{\star}$ & CCA-anonymity \\
\hline Our scheme & $O(n m \log q)$ & $O(n m \log q)$ & $O(t(m+\log N) \log q)^{\star \star}$ & CCA-anonymity \\
\hline
\end{tabular}

* $\beta=\omega(\sqrt{n \log q \log n}) \log m$ is the integer norm bound in [44].

** Since $N$ is always a polynomial in $n$ (thus in $m$ ), this term is actually bounded by $O(t m \log q)$. Besides, we note that group signatures supporting opening should have a signature in bit-size at least logarithmic in $N$ [11].

general paradigm and does not make use of any encryption. Although all three schemes use (almost) the same encryption (namely [57]), the NIZKs are very different. Concretely, Gordon et al. [37] used a $N$-OR variant of the witnessindistinguishable (WI) proof system for the gap version of the closest vector problem in [54], while the NIZKs used in [43] and ours are derived from the more efficient protocol [50] for the ISIS problem. In Table 2, we simply compare the complexity of each algorithm of the schemes in $[37,43]$ and ours, with respect to the number of basic operations in terms of encryptions and basic NIZKs.

Table 2. Rough Comparison of Computational Costs (The encryption and decryption of Regev's LWE-based encryption [57] are denoted by enc. and dec., respectively. The proof and verification of the corresponding basic NIZKs in [50,54] are denoted by pro. and ver., respectively).

\begin{tabular}{|c|c|c|c|}
\hline Schemes & Sign (enc.,pro.,ver.,dec.) & Verify (enc.,pro.,ver.,dec.) & Open (enc.,pro.,ver.,dec.) \\
\hline GKV10 [37] & $(N, O(N),-,-)$ & $(-,-, O(N),-)$ & $(-,-,-, N / 2)$ \\
\hline LLLS13 $[43]$ & $(1+\log N, O(\log N),-,-)$ & $(-,-, O(\log N),-)$ & $(-,-,-, 1+\log N)$ \\
\hline Our scheme & $(1, \leq 5,-,-)$ & $(-,-, \leq 5,-)$ & $(-,-,-, 1)$ \\
\hline
\end{tabular}

However, we note that we do not provide a full comparison with all the schemes in $[37,43,44]$, which would require at least a concrete analysis of the security reduction (running time, lattice approximation factor, success probability, etc.), which is usually not explicitly given in the literature.

\subsection{Techniques}

At a high level, the two constructions in $[37,43]$ and our scheme use the same general paradigm as that of [11]. Roughly speaking, the group manager first generates the group public key gpk and group manager secret key gmsk. For a user with identity $i \in\{1, \ldots, N\}$ (recall that $N$ is the maximum number of group members, and is fixed at the system setup), the group manager computes the user's secret key $g s k_{i}$ corresponding to an encoded "public key" $H(g p k, i)$, where $H$ is an encoding function that (uniquely) encodes the group user's identity $i$ in 
$g s k_{i}$. When signing a message $m$, the group user proves to the verifier that he has a secret key $g s k_{i}$ for some $i \in\{1, \ldots, N\}$ (i.e., to prove that he is a legal member of the group). The hardness of this general paradigm usually lies in the choices of an appropriate encoding function $H(g p k, i)$ and a compatible non-interactive zero-knowledge (NIZK) for the membership relations determined by $H(g p k, i)$.

Gordon et al. [37] used a simple projective encoding function $H(g p k, i)$ and a NIZK extended from [54] to construct the first lattice-based group signature. Informally, the group public key gpk consists of $N$ independent public keys of the GPV signature [36], i.e., $g p k=\left(p k_{1}, \ldots, p k_{N}\right)$ where $p k_{j}$ is an integer matrix over $\mathbb{Z}_{q}$ for some positive $q \in \mathbb{Z}$, and all $j \in\{1, \ldots, N\}$. The encoding function simply outputs the $i$-th element of $g p k$, i.e., $H(g p k, i):=p k_{i}$. Due to the particular choice of $H(g p k, i)$, both the group public key and the signature of [37] have a size linear in $N$.

At Asiacrypt '13, by using an efficient encoding function inspired by Boyen's lattice-based signature [19] and a NIZK derived from [50], Laguillaumie et al. [43] proposed a more efficient lattice-based group signature. Roughly speaking, the group public key gpk consists of $\ell=\lfloor\log N\rfloor+1$ independent matrices over $\mathbb{Z}_{q}$, i.e., gpk $=\left(\mathbf{A}_{1}, \ldots, \mathbf{A}_{\ell}\right)$. The encoding function is defined as $H(g p k, i):=$ $\sum_{j=1}^{\ell} i_{j} \mathbf{A}_{j}$, where $\left(i_{1}, \ldots, i_{l}\right) \in \mathbb{Z}_{2}^{\ell}$ is the binary decomposition of $i$. We also note that Langlois et al. [44] constructed a lattice-based group signature with verifierlocal revocation by using the same identity encoding function but a different NIZK from [47]. Both schemes [43,44] decreased the sizes of the group public key and the signature to proportional to $\log N$.

An Efficient Identity Encoding. We use a more efficient and compact way to encode the group member's identity, by building upon the encoding technique introduced by Agrawal et al. [2] for identity-based encryption (IBE). Let the group public key $g p k$ consist of three matrices over $\mathbb{Z}_{q}^{n \times m}$ for some positive integers $n, m, q$, i.e., gpk $=\left(\mathbf{A}_{1}, \mathbf{A}_{2,1}, \mathbf{A}_{2,2}\right)$. We define $H(g p k, i)=\hat{\mathbf{A}}_{i}:=$ $\left(\mathbf{A}_{1} \| \mathbf{A}_{2,1}+G(i) \mathbf{A}_{2,2}\right)$ where $G(\cdot)$ is a function from $\mathbb{Z}_{N}$ to $\mathbb{Z}_{q}^{n \times n}$. Then, the secret key of user $i$ is a short basis of the classical $q$-ary lattice $\boldsymbol{\Lambda}_{i}$ determined by $H(g p k, i)=\hat{\mathbf{A}}_{i}$, where $\boldsymbol{\Lambda}_{i}:=\left\{\mathbf{e} \in \mathbb{Z}^{m}\right.$ s.t. $\left.\hat{\mathbf{A}}_{i} \mathbf{e}=\mathbf{0} \bmod q\right\}$. When signing a message, user $i$ samples a short vector $\mathbf{e}_{i}$ from $\boldsymbol{\Lambda}_{i}$ (by using the short basis of $\boldsymbol{\Lambda}_{i}$ ) and encrypts it using Regev's encryption [57]. Then, he proves to the verifier that $\mathbf{e}_{i}$ is a short vector in a lattice determined by $H(g p k, i)$ for some $i \in\{1, \ldots, N\}$. But we do not know an efficient lattice-based NIZK suitable for the membership relation determined by $H(g p k, i)$.

Fortunately, since the maximum number of group members $N$ is always bounded by a polynomial in the security parameter $n$, we actually do not need an encoding function as powerful as for IBE [2], where there are possibly exponentially many users. We simplify the encoding function by defining $H(g p k, i):=\left(\mathbf{A}_{1} \| \mathbf{A}_{2,1}+i \mathbf{A}_{2,2}\right)$. (A similar combination of matrices has been used in a different way in $[4,17,35]$ to construct functional encryption.) Namely, the identity function $G(i):=i$ is used instead of a function $G: \mathbb{Z}_{N} \rightarrow \mathbb{Z}_{q}^{n \times n}$. For collision resistance, we require that $N<q$. Since $N$ is usually fixed at the system 
setup in group signatures for static groups such as [37,43,44] and ours, one can simply set $q$ big enough (but still a polynomial in $n$ ) to satisfy the requirement. Hereafter, we assume that $N<q$ always holds.

This encoding function provides two main benefits:

- Only three matrices are needed for the encoding function, which provides a short group public key. By comparison, there are respectively at least $O(N)$ and $O(\log N)$ matrices needed in [37] and [43].

- It gives a simple membership relation, which allows to construct an efficient NIZK proof for the relation (please see next paragraph). In $[37,43]$, the NIZKs for relatively complex membership relations are obtained by involving many encryptions, which results in schemes with large computational costs and signature sizes.

A New Non-interactive Zero-Knowledge (NIZK). Recall that the secret key of user $i$ is a short basis $\mathbf{T}_{i}$ of the $q$-ary lattice $\boldsymbol{\Lambda}_{i}$ determined by $\hat{\mathbf{A}}_{i}=\left(\mathbf{A}_{1} \| \mathbf{A}_{2,1}+\right.$ $\left.i \mathbf{A}_{2,2}\right)$. To sign a message, user $i$ first samples a short vector $\left(\mathbf{x}_{1}, \mathbf{x}_{2}\right)$ by using Gentry et al.'s Gaussian sampling algorithm [36] such that $\mathbf{A}_{1} \mathbf{x}_{1}+\left(\mathbf{A}_{2,1}+\right.$ $\left.i \mathbf{A}_{2,2}\right) \mathbf{x}_{2}=\mathbf{0} \bmod q$. Then, he generates an LWE encryption $\mathbf{c}$ of $\mathbf{x}_{1}$. The final signature $\sigma$ consists of $\mathbf{c}, \mathbf{x}_{2}$, a proof $\pi_{1}$ that $\mathbf{c}$ encrypts $\mathbf{x}_{1}$ correctly, and a proof $\pi_{2}$ that there exists a tuple $\left(\mathbf{x}_{1}, i\right)$ satisfying $\mathbf{A}_{1} \mathbf{x}_{1}+i \mathbf{A}_{2,2} \mathbf{x}_{2}=-\mathbf{A}_{2,1} \mathbf{x}_{2} \bmod q$, namely, $\sigma=\left(\mathbf{c}, \mathbf{x}_{2}, \pi_{1}, \pi_{2}\right)$.

The nice properties of the sampling algorithm in $[31,36]$ guarantee that the public $\mathbf{x}_{2}$ is statistically indistinguishable for all user $i \in\{1, \ldots, N\}$, namely, the verifier cannot determine the signer's identity $i$ solely from $\mathbf{x}_{2}$, however, he can efficiently determine it from $\left(\mathbf{x}_{1}, \mathbf{x}_{2}\right)$, that's why we choose to encrypt $\mathbf{x}_{1}$. The proof of $\pi_{1}$ can be generated by using the duality of LWE and Small Integer Solutions (SIS) [51], and the NIZK proof for SIS [50] in a standard way. Thanks to our new identity encoding function $H(g p k, i)$ and the public $\mathbf{x}_{2}$, we manage to design a NIZK proof (i.e., $\pi_{2}$ ) for the statement $\mathbf{A}_{1} \mathbf{x}_{1}+i \mathbf{A}_{2,2} \mathbf{x}_{2}=-\mathbf{A}_{2,1} \mathbf{x}_{2}$ $\bmod q$ based on the hardness of SIS.

Formally, we introduce a new problem called split-SIS, which is a variant of SIS (and might be of independent interest). Given a split-SIS instance $\mathbf{A}_{1}, \mathbf{A}_{2,2} \in$ $\mathbb{Z}_{q}^{n \times m}$, the algorithm is asked to output a triple $\left(\mathbf{x}_{1}, \mathbf{x}_{2}, h\right)$ such that $\mathbf{x}_{1}, \mathbf{x}_{2} \in$ $\mathbb{Z}^{m}$ have small norms, and $h<q=\operatorname{poly}(n)$ is a positive integer satisfying $\mathbf{A}_{1} \mathbf{x}_{1}+h \mathbf{A}_{2,2} \mathbf{x}_{2}=\mathbf{0} \bmod q$. We first show that the split-SIS problem (associated with an appropriate solution space) is polynomially equivalent to the standard SIS problem. Then, we derive a family of hash functions

$$
\mathcal{H}=\left\{\begin{array}{c}
f_{\mathbf{A}_{1}, \mathbf{A}_{2,2}}\left(\mathbf{x}_{1}, \mathbf{x}_{2}, h\right)=\left(\mathbf{A}_{1} \mathbf{x}_{1}+h \mathbf{A}_{2,2} \mathbf{x}_{2} \bmod q, \mathbf{x}_{2}\right): \\
\left(\mathbf{x}_{1}, \mathbf{x}_{2}, h\right) \in \mathbb{Z}^{m} \times \mathbb{Z}^{m} \times \mathbb{Z}
\end{array}\right\}_{\mathbf{A}_{1}, \mathbf{A}_{2,2} \in \mathbb{Z}_{q}^{n \times m}}
$$

from our split-SIS problem, and prove that the hash function family $\mathcal{H}$ with appropriate domain is one-way, collision-resistant, and statistically hiding with respect to the third input (i.e., $h$ ). Combining those useful properties with the observation that $\mathbf{A}_{1} \mathbf{x}_{1}+h \mathbf{A}_{2,2} \mathbf{x}_{2}=\left(\mathbf{A}_{1} \| \mathbf{A}_{2,2} \mathbf{x}_{2}\right)\left(\mathbf{x}_{1} ; h\right) \bmod q$, we manage 
to adapt a $\Sigma$-protocol for $\mathcal{H}$ from existing protocols for standard ISIS problems $[43,49,50]$, which can in turn be transformed into a NIZK using the FiatShamir transformation in the random oracle model. This finally helps us obtain a lattice-based group signature scheme with $O(t m \log q)$-bit signature, where the repetition parameter $t=\omega(\log n)$ is due to our NIZK as in $[43,44]$.

In order to open a signature $\sigma=\left(\mathbf{c}, \mathbf{x}_{2}, \pi_{1}, \pi_{2}\right)$, the group manager only has to decrypt $\mathbf{c}$ to obtain $\mathbf{x}_{1}$, and computes an integer $h<q$ satisfying $\mathbf{A}_{1} \mathbf{x}_{1}+$ $h \mathbf{A}_{2,2} \mathbf{x}_{2}=-\mathbf{A}_{2,1} \mathbf{x}_{2} \bmod q$. Note that such an integer is unique if $\mathbf{A}_{2,2} \mathbf{x}_{2} \neq \mathbf{0}$ $\bmod q$ for prime $q$. Replacing the CPA-encryption of $\mathbf{x}_{1}$ with a CCA one (i.e., by applying the CHK transformation [30] to the IBEs $[2,36]$ ), we obtain a CCAanonymous group signature at a minimal price of doubling the sizes of the group public key and the signature.

\subsection{On Membership Revocation}

A group signature with opening allows the group manager to break the anonymity of any valid signature, however, it cannot prevent a malicious group member from using his certificate. In practice, it may be desirable to support membership revocation, e.g., to revoke the certificate of a malicious group member such that he cannot sign any message in the future. Actually, membership revocation is an important and complex problem and has been extensively studied in the literature $[10,18,25,27,45,46]$. In [44], Langlois et al. constructed a lattice-based group signature with verifier-local revocation, which was the first lattice-based group signatures supporting membership revocation and achieved the same asymptotic efficiency as that of [43]. For now, we do not know how to construct a simpler and efficient group signature with membership revocation from lattices.

\subsection{Roadmap}

After some preliminaries, we recall several useful tools and algorithms on lattices in Section 3. In Section 4, we introduce the split-SIS problems, and construct a NIZK proof for the split-SIS problems. We finally present our CPA-anonymous group signature scheme in Section 5. The description of our CCA-anonymous group signature scheme is deferred to the full version.

\section{Preliminaries}

\section{$2.1 \quad$ Notation}

The set of real numbers (integers) is denoted by $\mathbb{R}$ ( $\mathbb{Z}$, resp.). By $\leftarrow{ }_{R}$ we denote randomly choosing elements from some distribution (or the uniform distribution over some finite set). For a variable $x$ following some distribution $D$, we denote it by $x \backsim D$. For any integer $N \in \mathbb{Z}$, we denote by $[N]$ the set of integers $\{0,1, \ldots, N-1\}$. Vectors are in column form and denoted by bold lower-case letters $(e . g ., \mathbf{x})$. We view a matrix simply as the set of its column vectors and 
denoted by bold capital letters $(e . g ., \mathbf{X})$. Denote the $l_{2}$ and $l_{\infty}$ norm by $\|\cdot\|$ and $\|\cdot\|_{\infty}$, respectively. Define the norm of a matrix $\mathbf{X}$ as the norm of its longest column (i.e., $\left.\|\mathbf{X}\|=\max _{i}\left\|\mathbf{x}_{i}\right\|\right)$. If the columns of $\mathbf{X}=\left(\mathbf{x}_{1}, \ldots, \mathbf{x}_{k}\right)$ are linearly independent, let $\widetilde{\mathbf{X}}=\left(\widetilde{\mathbf{x}}_{1}, \ldots, \widetilde{\mathbf{x}}_{k}\right)$ denote the Gram-Schmidt orthogonalization of vectors $\mathbf{x}_{1}, \ldots, \mathbf{x}_{k}$ taken in that order. For $\mathbf{X} \in \mathbb{R}^{n \times m}$ and $\mathbf{Y} \in \mathbb{R}^{n \times m^{\prime}}$, $(\mathbf{X} \| \mathbf{Y}) \in \mathbb{R}^{n \times\left(m+m^{\prime}\right)}$ denotes the concatenation of the columns of $\mathbf{X}$ followed by the columns of $\mathbf{Y}$. Similarly, for $\mathbf{X} \in \mathbb{R}^{n \times m}$ and $\mathbf{Y} \in \mathbb{R}^{n^{\prime} \times m},(\mathbf{X} ; \mathbf{Y}) \in \mathbb{R}^{\left(n+n^{\prime}\right) \times m}$ is the concatenation of the rows of $\mathbf{X}$ followed by the rows of $\mathbf{Y}$.

Throughout this paper, we let $n$ be the natural security parameter, so that all quantities are implicitly dependent on $n$. The function log denotes the natural logarithm. We will frequently use the standard notation of $O, \omega$ for classifying the growth of functions. If $f(n)=O\left(g(n) \cdot \log ^{c}(n)\right)$ for some constant $c$, we write $f(n)=\tilde{O}(g(n))$. By poly $(n)$ we denote some arbitrary $f(n)=O\left(n^{c}\right)$ for some $c$. We say that a function $f(n)$ is negligible if for every positive $c$, we have $f(n)<n^{-c}$ for sufficiently large $n$. We denote an arbitrary such function by $n e g l(n)$, and say that a probability is overwhelming if it is $1-n e g l(n)$.

\subsection{Group Signatures}

We recall the definition and security model of group signatures. A (static) group signature scheme $\mathcal{G S}$ consists of a tuple of four Probabilistic Polynomial Time (PPT) algorithms (KeyGen, Sign, Verify, Open):

- KeyGen $\left(1^{n}, 1^{N}\right)$ : Take the security parameter $n$ and the maximum number of group members $N$ as inputs, output the group public key gpk, the group manager secret key gmsk and a vector of users' keys gsk $=\left(g s k_{1}, \ldots, g s k_{N}\right)$, where $g s k_{j}$ is the $j$-th user's secret key for $j \in\{1, \ldots, N\}$.

- Sign $\left(g p k, g s k_{j}, M\right)$ : Take the group public key gpk, the $j$-th user's secret key $g s k_{j}$, and a message $M \in\{0,1\}^{*}$ as inputs, output a signature $\sigma$ of $M$.

- Verify $(g p k, M, \sigma)$ : Take the group public key gpk, a message $M \in\{0,1\}^{*}$ and a string $\sigma$ as inputs, return 1 if $\sigma$ is a valid signature of $M$, else return 0 .

- Open $(g p k, g m s k, M, \sigma)$ : Take the group public key $g p k$, the group manager secret key gmsk, a message $M \in\{0,1\}^{*}$, and a valid signature $\sigma$ of $M$ as inputs, output an index $j \in\{1, \ldots, N\}$ or a special symbol $\perp$ in case of opening failure.

For correctness, we require that for any $(g p k, g m s k$, gsk $) \leftarrow \operatorname{KeyGen}\left(1^{n}, 1^{N}\right)$, any $j \in\{1, \ldots, N\}$, any message $M \in\{0,1\}^{*}$, and any $\sigma \leftarrow \operatorname{Sign}\left(g p k, g s k_{j}, M\right)$, the following conditions hold with overwhelming probability:

$$
\operatorname{Verify}(g p k, M, \sigma)=1 \text { and Open }(g p k, g m s k, M, \sigma)=j
$$

For group signatures, there are two security notions: anonymity and traceability [11]. The first notion, informally, says that anyone without the group manager secret key cannot determine the owner of a valid signature. The second 
notion says a set $\mathcal{C}$ of group members cannot collude to create a valid signature such that the Open algorithm fails to trace back to one of them. In particular, this notion implies that any non-group member cannot create a valid signature.

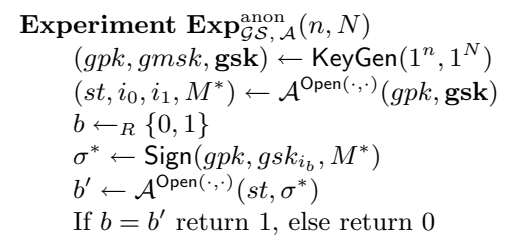

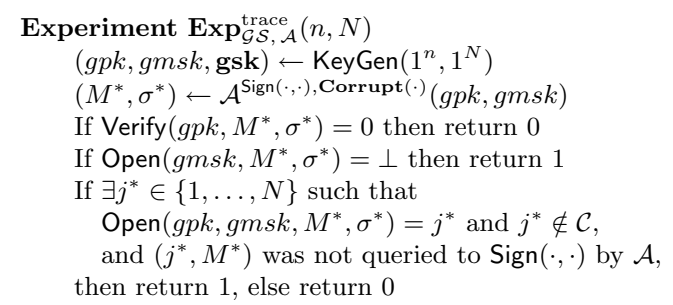

Fig. 1. Security games for group signatures

Definition 1 (Full anonymity). For any (static) group signature scheme $\mathcal{G S}$, we associate to an adversary $\mathcal{A}$ against the full anonymity of $\mathcal{G} \mathcal{S}$ experiment $\operatorname{Exp}_{\mathcal{G} \mathcal{S}, \mathcal{A}}^{\text {anon }}(n, N)$ in the left-side of Fig. 1, where the Open $(\cdot, \cdot)$ oracle takes a valid message-signature pair $(M, \sigma)$ as inputs, outputs the index of the user whose secret key is used to create $\sigma$. In the guess phase, the adversary $\mathcal{A}$ is not allowed to make an Open query with inputs $\left(M^{*}, \sigma^{*}\right)$. We define the advantage of $\mathcal{A}$ in the experiment as

$$
\operatorname{Adv}_{\mathcal{G} \mathcal{S}, \mathcal{A}}^{\text {anon }}(n, N)=\left|\operatorname{Pr}\left[\operatorname{Exp}_{\mathcal{G S}, \mathcal{A}}^{\text {anon }}(n, N)=1\right]-\frac{1}{2}\right| .
$$

A group signature $\mathcal{G S}$ is said to be fully anonymous if the advantage $\operatorname{Adv}_{\mathcal{G S}, \mathcal{A}}^{\text {anon }}(n$, $N)$ is negligible in $n, N$ for any PPT adversary $\mathcal{A}$.

In a weak definition of anonymity (i.e., CPA-anonymity), the adversary is not given access to an open oracle. In this paper, we first present a CPA-anonymous scheme, then we extend it to satisfy full/CCA anonymity.

Definition 2 (Full traceability). For any (static) group signature scheme $\mathcal{G S}$, we associate to an adversary $\mathcal{A}$ against the full traceability of $\mathcal{G S}$ experiment $\operatorname{Exp}_{\mathcal{G} \mathcal{S}, \mathcal{A}}^{\text {trace }}(n, N)$ in the right-side of Fig. 1, where the Sign $(\cdot, \cdot)$ oracle takes a user index $i$ and a message $M$ as inputs, returns a signature of $M$ by using gsk $_{i}$. The Corrupt(·) oracle takes a user index $i$ as input, returns gs $k_{i}$, and $\mathcal{C}$ is a set of user indexes that $\mathcal{A}$ submitted to the Corrupt $(\cdot)$ oracle. The advantage of $\mathcal{A}$ in the experiment is defined as

$$
\operatorname{Adv}_{\mathcal{G} \mathcal{S}, \mathcal{A}}^{\text {trace }}(n, N)=\operatorname{Pr}\left[\operatorname{Exp}_{\mathcal{G} \mathcal{S}, \mathcal{A}}^{\text {trace }}(n, N)=1\right]
$$

A group signature $\mathcal{G S}$ is said to be fully traceable if the advantage $\operatorname{Adv}_{\mathcal{G} \mathcal{S}, \mathcal{A}}^{\operatorname{trace}}(n, N)$ is negligible in $n, N$ for any PPT adversary $\mathcal{A}$. 


\section{Lattices and Discrete Gaussians}

An $m$-rank lattice $\boldsymbol{\Lambda} \subset \mathbb{R}^{n}$ is the set of all integral combinations of $m$ linearly independent vectors $\mathbf{B}=\left(\mathbf{b}_{1}, \ldots, \mathbf{b}_{m}\right) \in \mathbb{R}^{n \times m}$, i.e., $\boldsymbol{\Lambda}=\mathcal{L}(\mathbf{B})=\left\{\sum_{i=1}^{m} x_{i} \mathbf{b}_{i}\right.$ : $\left.x_{i} \in \mathbb{Z}\right\}$. The dual lattice of $\boldsymbol{\Lambda}$ is defined to be $\boldsymbol{\Lambda}^{*}=\{\mathbf{x} \in \operatorname{span}(\Lambda): \forall \mathbf{v} \in$ $\Lambda,\langle\mathbf{x}, \mathbf{v}\rangle \in \mathbb{Z}\}$.

For $\mathbf{x} \in \boldsymbol{\Lambda}$, define the Gaussian function $\rho_{s, \mathbf{c}}(\mathbf{x})$ over $\boldsymbol{\Lambda} \subseteq \mathbb{Z}^{n}$ centered at $\mathbf{c} \in \mathbb{R}^{n}$ with parameter $s>0$ as $\rho_{s, \mathbf{c}}(\mathbf{x})=\exp \left(-\pi\|\mathbf{x}-\mathbf{c}\|^{2} / s^{2}\right)$. Letting $\rho_{s, \mathbf{c}}(\boldsymbol{\Lambda})=\sum_{\mathbf{x} \in \boldsymbol{\Lambda}} \rho_{s, \mathbf{c}}(\mathbf{x})$, define the discrete Gaussian distribution over $\boldsymbol{\Lambda}$ as $D_{\boldsymbol{\Lambda}, s, \mathbf{c}}(\mathbf{y})=\frac{\rho_{s, \mathbf{c}}(\mathbf{y})}{\rho_{s, \mathbf{c}}(\boldsymbol{\Lambda})}$, where $\mathbf{y} \in \boldsymbol{\Lambda}$. The subscripts $s$ and $\mathbf{c}$ are taken to be 1 and $\mathbf{0}$ (respectively) when omitted. For large enough $s$, almost all the elements from $D_{\boldsymbol{\Lambda}, s, \mathbf{c}}$ are not far from $\mathbf{c}$.

Lemma 1 ([36,53]). For any $n$-dimensional lattice $\boldsymbol{\Lambda}$ with basis $\mathbf{B} \in \mathbb{R}^{n \times n}$, vector $\mathbf{c} \in \mathbb{R}^{n}$, and reals $\epsilon \in(0,1), s \geq\|\widetilde{\mathbf{B}}\| \cdot \omega(\sqrt{\log n})$, we have $\operatorname{Pr}_{\mathbf{x} \leftarrow{ }_{R} D_{\boldsymbol{\Lambda}, s, \mathbf{c}}}[\| \mathbf{x}-$ $\mathbf{c} \|>s \sqrt{n} \leq \frac{1-\epsilon}{1+\epsilon} \cdot 2^{-n}$.

For any $\alpha \in \mathbb{R}^{+}$, integer $q \in \mathbb{Z}$, let $\Psi_{\alpha}$ be the distribution over $\mathbb{T}=\mathbb{R} / \mathbb{Z}$ of a normal variable with mean 0 and standard deviation $\alpha / \sqrt{2 \pi}$, reduced modulo 1. The discrete distribution $\bar{\Psi}_{\alpha}$ over $\mathbb{Z}_{q}$ is the random variable $\lfloor q \cdot X\rceil \bmod q$, where $X \leftarrow_{R} \Psi_{\alpha}$. For simplicity, we denote $\chi_{\alpha}:=\bar{\Psi}_{\alpha}$.

Lemma 2 ([3]). Let $\mathbf{e}$ be some vector in $\mathbb{Z}^{m}$ and let $\mathbf{y} \leftarrow_{R} \chi_{\alpha}^{m}$. Then the quantity $\left|\mathbf{e}^{T} \mathbf{y}\right|$ treated as an integer in $[0, q-1]$ satisfies $\left|\mathbf{e}^{T} \mathbf{y}\right| \leq\|\mathbf{e}\| q \alpha \omega(\sqrt{\log m})+$ $\|\mathbf{e}\| \sqrt{m} / 2$ with all but negligible probability in $m$. In particular, if $x \leftarrow_{R} \chi_{\alpha}$ is treated as an integer in $[0, q-1]$ then $|x| \leq q \alpha \omega(\sqrt{\log m})+1 / 2$ with all but negligible probability in $m$.

We also need the following three useful facts from the literature:

Lemma 3 ([36]). Let $n$ be a positive integer, $q$ be a prime, and $m \geq 2 n \log q$. Then for all but a $2 q^{-n}$ fraction of all $\mathbf{A} \in \mathbb{Z}_{q}^{n \times m}$ and for any $s \geq \omega(\sqrt{\log m})$, the distribution of $\mathbf{u}=\mathbf{A e} \bmod q$ is statistically close to uniform over $\mathbb{Z}_{q}^{n}$, where $\mathbf{e} \leftarrow{ }_{R} D_{\mathbb{Z}^{m}, s}$.

\subsection{Learning with Errors (LWE) and Small Integer Solutions (SIS)}

Let $n \in \mathbb{Z}^{+}$and $q=q(n)$ be integers, $\alpha \in \mathbb{R}^{+}, \chi_{\alpha}$ be some discrete Gaussian distribution over $\mathbb{Z}_{q}$, and $\mathbf{s} \in \mathbb{Z}_{q}^{n}$ be some vector. Define $A_{\mathbf{s}, \chi_{\alpha}} \subseteq \mathbb{Z}_{q}^{n} \times \mathbb{Z}_{q}$ as the distribution of the variable $\left(\mathbf{a}, \mathbf{a}^{T} \mathbf{s}+x\right)$, where $\mathbf{a} \leftarrow_{R} \mathbb{Z}_{q}^{n}, x \leftarrow_{R} \chi_{\alpha}$, and all the operations are performed in $\mathbb{Z}_{q}$. For $m$ independent samples $\left(\mathbf{a}_{1}, y_{1}\right), \ldots,\left(\mathbf{a}_{m}, y_{m}\right)$ from $A_{\mathbf{s}, \chi_{\alpha}}$, we denote it in matrix form $(\mathbf{A}, \mathbf{y}) \in \mathbb{Z}_{q}^{n \times m} \times \mathbb{Z}_{q}^{m}$, where $\mathbf{A}=$ $\left(\mathbf{a}_{1}, \ldots, \mathbf{a}_{m}\right)$ and $\mathbf{y}=\left(y_{1}, \ldots, y_{m}\right)^{T}$. We say that an algorithm solves $\mathrm{LWE}_{q, \chi_{\alpha}}$ if, for randomly chosen $\mathbf{s} \in \mathbb{Z}_{q}^{n}$, given polynomial samples from $\mathrm{A}_{\mathbf{s}, \chi_{\alpha}}$ it outputs 
s with overwhelming probability. The decisional variant of LWE is that, for a uniformly chosen $\mathbf{s} \leftarrow R \mathbb{Z}_{q}^{n}$, an algorithm is asked to distinguish $A_{\mathbf{s}, \chi_{\alpha}}$ from the uniform distribution over $\mathbb{Z}_{q}^{n} \times \mathbb{Z}_{q}$ (with only polynomial samples). For certain modulus $q$, the average-case decisional LWE problem is polynomially equivalent to its worst-case search version $[8,55,57]$.

Proposition 1 ([57]). Let $\alpha=\alpha(n) \in(0,1)$ and let $q=q(n)$ be a prime such that $\alpha q>2 \sqrt{n}$. If there exists an efficient (possibly quantum) algorithm that solves $L W E_{q, \chi_{\alpha}}$, then there exists an efficient quantum algorithm for approximating SIVP in the $l_{2}$ norm, in the worst case, to within $\tilde{O}(n / \alpha)$ factors.

The Small Integer Solution (SIS) problem was introduced by Ajtai [5], but its name is due to Micciancio and Regev [53], who improved Ajtai's connection between SIS and worst-case lattice problems.

Definition 3 (Small Integer Solution). The Small Integer Solution (SIS) problem in $l_{2}$ norm is: Given an integer $q$, a uniformly random matrix $\mathbf{A} \in$ $\mathbb{Z}_{q}^{n \times m}$, and a real $\beta$, find a non-zero integer vector $\mathbf{e} \in \mathbb{Z}^{m}$ such that $\mathbf{A e}=\mathbf{0}$ $(\bmod q)$ and $\|\mathbf{e}\| \leq \beta$.

Definition 4 (Inhomogeneous Small Integer Solution). The Inhomogeneous Small Integer Solution (ISIS) problem in $l_{2}$ norm is: Given an integer $q$, a uniformly random matrix $\mathbf{A} \in \mathbb{Z}_{q}^{n \times m}$, a random syndrome $\mathbf{u} \in \mathbb{Z}_{q}^{n}$, and real $\beta \in \mathbb{R}$, find an integer vector $\mathbf{e} \in \mathbb{Z}^{m}$ such that $\mathbf{A e}=\mathbf{u}(\bmod q)$ and $\|\mathbf{e}\| \leq \beta$.

The ISIS problem is an inhomogenous variant of SIS. Both problems were shown to be as hard as certain worst-case lattice problems.

Proposition 2 ([36]). For any polynomially bounded $m, \beta=\operatorname{poly}(n)$ and prime $q \geq \beta \cdot \omega(\sqrt{n \log n})$, the average-case problems $S I S_{q, m, \beta}$ and $I S I S_{q, m, \beta}$ are as hard as approximating SIVP in the worst case to within certain $\gamma=\beta \cdot \widetilde{O}(\sqrt{n})$ factors.

\section{2 $q$-ary Lattices and Trapdoors}

Let $\mathbf{A} \in \mathbb{Z}_{q}^{n \times m}$ for some positive integers $n, m$ and $q$. Consider the following two integer lattices:

$$
\begin{gathered}
\boldsymbol{\Lambda}_{q}^{\perp}(\mathbf{A})=\left\{\mathbf{e} \in \mathbb{Z}^{m} \text { s.t. } \mathbf{A e}=0 \bmod q\right\} \\
\boldsymbol{\Lambda}_{q}(\mathbf{A})=\left\{\mathbf{y} \in \mathbb{Z}^{m} \text { s.t. } \exists \mathbf{s} \in \mathbb{Z}^{n}, \mathbf{A}^{\mathbf{T}} \mathbf{s}=\mathbf{y} \bmod q\right\}
\end{gathered}
$$

The two $q$-ary lattices defined above are dual when properly scaled, namely $\boldsymbol{\Lambda}_{q}^{\perp}(\mathbf{A})=q \boldsymbol{\Lambda}_{q}(\mathbf{A})^{*}$ and $\boldsymbol{\Lambda}_{q}(\mathbf{A})=q \boldsymbol{\Lambda}_{q}^{\perp}(\mathbf{A})^{*}$. Moreover, for any $h \in \mathbb{Z}_{q}^{*}$, we have: $\boldsymbol{\Lambda}_{q}^{\perp}(\mathbf{A})=\boldsymbol{\Lambda}_{q}^{\perp}(h \mathbf{A})$.

In 1999, Ajtai [6] showed how to sample an essentially uniform matrix $\mathbf{A}$ together with a short basis of $\boldsymbol{\Lambda}_{q}^{\perp}(\mathbf{A})$. This trapdoor generation algorithm has been significantly improved in $[7,52]$. 
Proposition 3 ([7]). For any $\delta_{0}>0$, there is a PPT algorithm TrapGen that, on input a security parameter $n$, an odd prime $q=$ poly $(n)$, and integer $m \geq$ $\left(5+3 \delta_{0}\right) n \log q$, outputs a statistically $\left(m q^{-\delta_{0} n / 2}\right)$-close to uniform matrix $\mathbf{A} \in$ $\mathbb{Z}_{q}^{n \times m}$ and a basis $\mathbf{T}_{\mathbf{A}} \subset \boldsymbol{\Lambda}_{q}^{\perp}(\mathbf{A})$ such that with overwhelming probability $\left\|\mathbf{T}_{\mathbf{A}}\right\| \leq$ $O(n \log q)$ and $\left\|\widetilde{\mathbf{T}}_{\mathbf{A}}\right\| \leq O(\sqrt{n \log q})=O(\sqrt{m})$. In particular, if let $\delta_{0}=\frac{1}{3}$, we can choose $m \geq\lceil 6 n \log q\rceil$.

The following proposition is implied by [31, Lem.3.2andLem.3.3] which shows that there is an efficient algorithm to extract a random basis for $(\mathbf{A} \| \mathbf{B})$ by using a short basis of $\mathbf{A}$ such that the new basis statistically hides the information of its input basis.

Proposition 4 ([31]). There is a PPT algorithm ExtRndBasis which takes a matrix $\mathbf{A}^{\prime}=(\mathbf{A} \| \mathbf{B}) \in \mathbb{Z}_{q}^{n \times\left(m+m^{\prime}\right)}$, a basis $\mathbf{T}_{\mathbf{A}} \in \mathbb{Z}_{q}^{m \times m}$ of $\boldsymbol{\Lambda}_{q}^{\perp}(\mathbf{A})$, an arbitrary matrix $\mathbf{B} \in \mathbb{Z}_{q}^{n \times m^{\prime}}$, and a real $s \geq\left\|\widetilde{\mathbf{T}}_{\mathbf{A}}\right\| \cdot \omega(\sqrt{\log m})$ as inputs, outputs a random basis $\mathbf{T}_{\mathbf{A}^{\prime}}$ of $\boldsymbol{\Lambda}_{q}^{\perp}\left(\mathbf{A}^{\prime}\right)$ satisfying $\left\|\mathbf{T}_{\mathbf{A}^{\prime}}\right\| \leq s\left(m+m^{\prime}\right)$ and $\left\|\widetilde{\mathbf{T}}_{\mathbf{A}^{\prime}}\right\| \leq s \sqrt{m+m^{\prime}}$.

Equipped with the above proposition, and the proof technique of [2, Th.4], we obtain the following useful proposition:

Proposition 5. Let $q>2, m>n$, there is a PPT algorithm ExtBasisRight which takes matrix $\mathbf{A}^{\prime}=(\mathbf{C}\|\mathbf{A}\| \mathbf{A R}+\mathbf{B}) \in \mathbb{Z}_{q}^{n \times\left(2 m+m^{\prime}\right)}$, a uniformly and randomly chosen $\mathbf{R} \in\{-1,1\}^{m \times m}$, a basis $\mathbf{T}_{\mathbf{B}}$ of $\boldsymbol{\Lambda}_{q}^{\perp}(\mathbf{B})$, arbitrary $\mathbf{C} \in \mathbb{Z}_{q}^{n \times m^{\prime}}$ and a Gaussian parameter $s>\left\|\widetilde{\mathbf{T}}_{\mathbf{B}}\right\| \cdot \sqrt{m} \omega(\log m)$, outputs a basis $\mathbf{T}_{\mathbf{A}^{\prime}}$ of $\boldsymbol{\Lambda}_{q}^{\perp}\left(\mathbf{A}^{\prime}\right)$ satisfying $\left\|\mathbf{T}_{\mathbf{A}^{\prime}}\right\| \leq s\left(2 m+m^{\prime}\right)$ and $\left\|\widetilde{\mathbf{T}}_{\mathbf{A}^{\prime}}\right\| \leq s \sqrt{2 m+m^{\prime}}$.

Proof. As shown in the proof of [2, Th.4], we can use $\mathbf{T}_{\mathbf{B}}$ to efficiently sample a basis $\mathbf{T}_{\hat{\mathbf{A}}}$ for the matrix $\hat{\mathbf{A}}=(\mathbf{A} \| \mathbf{A R}+\mathbf{B})$ satisfying $\left\|\widetilde{\mathbf{T}}_{\hat{\mathbf{A}}}\right\| \leq$ $\left\|\widetilde{\mathbf{T}}_{\mathbf{B}}\right\| \cdot \sqrt{m} \omega(\sqrt{\log m})$, then we can apply Proposition 4 to obtain a basis $\mathbf{T}_{\mathbf{A}^{\prime}}$ for $\mathbf{A}^{\prime}=(\mathbf{C} \| \hat{\mathbf{A}})$ satisfying $\left\|\widetilde{\mathbf{T}}_{\mathbf{A}^{\prime}}\right\| \leq s \sqrt{2 m+m^{\prime}}$. Besides, by the property of the ExtRndBasis algorithm, the claim still holds no matter how the (columns of) matrix $\mathbf{C}$ appears in $\mathbf{A}^{\prime}$.

The following SuperSamp algorithm allows us to sample a random matrix $\mathbf{B}$ together with a short basis such that the columns of $\mathbf{B}$ lie in a prescribed affine subspace of $\mathbb{Z}_{q}^{n}$.

Proposition 6 ([43]). Let $q>2, m>\lceil 6 n \log q+n\rceil$, there is a PPT algorithm SuperSamp which takes matrices $\mathbf{A} \in \mathbb{Z}_{q}^{n \times m}$ and $\mathbf{C} \in \mathbb{Z}_{q}^{n \times n}$ as inputs, and outputs an almost uniform matrix $\mathbf{B} \in \mathbb{Z}_{q}^{n \times m}$ such that $\mathbf{A B}^{T}=\mathbf{C}$, and a basis $\mathbf{T}_{\mathbf{B}}$ of $\boldsymbol{\Lambda}_{q}^{\perp}(\mathbf{B})$ satisfying $\left\|\mathbf{T}_{B}\right\| \leq m^{1.5} \cdot \omega(\sqrt{\log m})$ and $\left\|\widetilde{\mathbf{T}}_{B}\right\| \leq m \cdot \omega(\sqrt{\log m})$.

Given a basis of $\boldsymbol{\Lambda}_{q}^{\perp}(\mathbf{A})$, there is an efficient algorithm to solve the (I)SIS problem as follows.

Proposition 7 ([36]). There is a PPT algorithm SamplePre that, given a basis $\mathbf{T}_{\mathbf{A}}$ of $\boldsymbol{\Lambda}_{q}^{\perp}(\mathbf{A})$, a real $s \geq\left\|\widetilde{\mathbf{T}}_{\mathbf{A}}\right\| \cdot \omega(\sqrt{\log m})$ and a vector $\mathbf{u} \in \mathbb{Z}_{q}^{n}$, outputs a vector $\mathbf{e} \sim D_{\mathbb{Z}^{m}, s}$ satisfying $\mathbf{A e}=\mathbf{u}$. 


\subsection{Non-interactive Zero-Knowledge Proofs of Knowledge}

In 2013, Laguillaumie et al. [43] adapted the protocol of [49,50] to obtain a zero-knowledge proof of knowledge for the ISIS problem in the random oracle model. Concretely, there is a non-interactive zero-knowledge proof of knowledge (NIZKPoK) for the ISIS relations

$$
R_{\mathrm{ISIS}}=\left\{(\mathbf{A}, \mathbf{y}, \beta ; \mathbf{x}) \in \mathbb{Z}_{q}^{n \times m} \times \mathbb{Z}_{q}^{n} \times \mathbb{R} \times \mathbb{Z}^{m}: \mathbf{A x}=\mathbf{y} \text { and }\|\mathbf{x}\| \leq \beta\right\} .
$$

In particular, there is a knowledge extractor which, given two valid proofs with the same commitment message but two different challenges, outputs a witness $\mathbf{x}^{\prime}$ satisfying $\left\|\mathbf{x}^{\prime}\right\| \leq O\left(\beta m^{2}\right)$ and $\mathbf{A} \mathbf{x}^{\prime}=\mathbf{y}$. By using the duality between LWE and ISIS, there exists an NIZKPoK for the LWE relation:

$$
R_{\mathrm{LWE}}=\left\{(\mathbf{A}, \mathbf{b}, \alpha ; \mathbf{s}) \in \mathbb{Z}_{q}^{n \times m} \times \mathbb{Z}_{q}^{m} \times \mathbb{R} \times \mathbb{Z}_{q}^{n}:\left\|\mathbf{b}-\mathbf{A}^{T} \mathbf{s}\right\| \leq \alpha q \sqrt{m}\right\} .
$$

Actually, as noted in [51], given a random matrix $\mathbf{A} \in \mathbb{Z}_{q}^{n \times m}$ such that the columns of $\mathbf{A}$ generate $\mathbb{Z}_{q}^{n}$ (this holds with overwhelming probability for a uniformly random $\left.\mathbf{A} \in \mathbb{Z}_{q}^{n \times m}\right)$, one can compute a matrix $\mathbf{G} \in \mathbb{Z}_{q}^{(m-n) \times m}$ such that 1) the columns of $\mathbf{G}$ generate $\left.\mathbb{Z}_{q}^{m-n} ; 2\right) \mathbf{G A}^{T}=\mathbf{0}$. Thus, to prove $(\mathbf{A}, \mathbf{b}, \alpha ; \mathbf{s}) \in$ $R_{\mathrm{LWE}}$, one can instead prove the existence of $\mathbf{e}$ such that $\|\mathbf{e}\| \leq \alpha q \sqrt{m}$ and $\mathbf{G e}=\mathbf{G b}$. In particular, in the construction of our group signature we need to prove that for given $(\mathbf{A}, \mathbf{b}) \in \mathbb{Z}_{q}^{n \times m} \times \mathbb{Z}_{q}^{m}$, there exist short vectors $(\mathbf{e}, \mathbf{x})$ such that $\|\mathbf{e}\| \leq \alpha q \sqrt{m},\|\mathbf{x}\| \leq \beta$ and $\mathbf{b}=\mathbf{A}^{T} \mathbf{s}+p \mathbf{e}+\mathbf{x}$ for some $\mathbf{s} \in \mathbb{Z}_{q}^{n}$, where $p \geq(\alpha q \sqrt{m}+\beta) m^{2}$. Similarly, this can also be achieved by proving the existence of short vectors $\mathbf{e}$ and $\mathbf{x}$ such that $p \mathbf{G e}+\mathbf{G x}=\mathbf{G b}$ using the NIZKPoK for ISIS relations. Formally, denoting $\gamma=\max (\alpha q \sqrt{m}, \beta)$, there exists an NIZKPoK for the extended-LWE (eLWE) relations

$$
\begin{aligned}
R_{\mathrm{eLWE}}=\left\{(\mathbf{A}, \mathbf{b}, \gamma ; \mathbf{s}, \mathbf{e}, \mathbf{x}) \in \mathbb{Z}_{q}^{n \times m} \times \mathbb{Z}_{q}^{m} \times \mathbb{R} \times \mathbb{Z}_{q}^{n} \times \mathbb{Z}^{2 m}:\right. \\
\left.\mathbf{b}=\mathbf{A}^{T} \mathbf{s}+p \mathbf{e}+\mathbf{x} \text { and }\|\mathbf{e}\| \leq \gamma \text { and }\|\mathbf{x}\| \leq \gamma\right\} .
\end{aligned}
$$

\section{Split-SIS Problems}

Given uniformly random matrices $\left(\mathbf{A}_{1}, \mathbf{A}_{2}\right) \in \mathbb{Z}_{q}^{n \times m} \times \mathbb{Z}_{q}^{n \times m}$, integer $N=N(n)$ and $\beta=\beta(n)$, an algorithm solving the split-SIS $\mathbf{S}_{q, m, \beta, N}$ problem is asked to output a tuple $\left(\mathbf{x}=\left(\mathbf{x}_{1} ; \mathbf{x}_{2}\right), h\right) \in \mathbb{Z}^{2 m} \times \mathbb{Z}$ such that

$-\mathbf{x}_{1} \neq 0$ or $h \mathbf{x}_{2} \neq 0$

- $\|\mathbf{x}\| \leq \beta, h \in[N]$, and $\mathbf{A}_{1} \mathbf{x}_{1}+h \mathbf{A}_{2} \mathbf{x}_{2}=0$.

Recall that the standard SIS $_{q, m^{\prime}, \beta}$ problem asks an algorithm to find a root of the hash function $f_{\mathbf{A}}(\mathbf{x})=\mathbf{A x}=\mathbf{0} \bmod q$ for a uniformly chosen matrix $\mathbf{A}$ and a "narrow" domain $\hat{D}_{m^{\prime}, \beta}:=\left\{\mathbf{x} \in \mathbb{Z}^{m^{\prime}}:\|\mathbf{x}\| \leq \beta\right\}$. While for the split$\mathrm{SIS}_{q, m, \beta, N}$ problem, the algorithm is allowed to "modify" the function by defining $f_{\mathbf{A}^{\prime}}\left(\mathbf{x}^{\prime}\right)=\mathbf{A}^{\prime} \mathbf{x}^{\prime}$ for $\mathbf{A}^{\prime}=\left(\mathbf{A}_{1} \| \mathbf{A}_{2} \mathbf{x}_{2}\right)$ with arbitrarily $\mathbf{x}_{2} \in \hat{D}_{m, \beta}$, and outputs a root $\mathbf{x}^{\prime}=\left(\mathbf{x}_{1}, h\right) \in \hat{D}_{m, \beta} \times[N]$. Intuitively, the split-SIS $q, m, \beta, N$ problem is 
not harder than $\operatorname{SIS}_{q, 2 m, \beta}$ problem. Since if $\mathbf{x}=\left(\mathbf{x}_{1}, \mathbf{x}_{2}\right)$ is a solution of the $\mathrm{SIS}_{q, 2 m, \beta}$ instance $\mathbf{A}=\left(\mathbf{A}_{1} \| \mathbf{A}_{2}\right),(\mathbf{x}, 1)$ is a solution of the split-SIS $q, m, \beta, N$ instance $\left(\mathbf{A}_{1}, \mathbf{A}_{2}\right)$ with $N \geq 1$.

However, for prime $q=q(n)$, and $N=N(n)<q$ of a polynomial in $n$, we show in the following theorem that the split-SIS $\operatorname{Sin}, \beta, N_{\text {problem is at least as }}$ hard as $\mathrm{SIS}_{q, 2 m, \beta}$. Thus, the average-case hardness of the split-SIS problem is based on the worst-case hardness of SIVP by Proposition 2 .

Theorem 1 (Hardness of Split-SIS Problems). For any polynomial $m=$ $m(n), \beta=\beta(n), N=N(n)$, and any prime $q \geq \beta \cdot \omega(\sqrt{n \log n})>N$, the split$S I S_{q, m, \beta, N}$ problem is polynomially equivalent to $S I S_{q, 2 m, \beta}$ problem. In particular, the average-case split-SIS $S_{q, m, \beta, N}$ is as hard as approximating the SIVP problem in the worst case to within certain $\gamma=\beta \cdot \widetilde{O}(\sqrt{n})$ factors.

Proof. The direction from split-SIS $q, m, \beta, N$ to $\operatorname{SIS}_{q, 2 m, \beta}$ is obvious. We now prove the other direction. Assume that there is an algorithm $\mathcal{A}$ that solves split-SIS ${ }_{q, m, \beta, N}$ with probability $\epsilon$, we now construct an algorithm $\mathcal{B}$ that solves $\mathrm{SIS}_{q, 2 m, \beta}$ with probability at least $\epsilon / N$ (recall that $N$ is a polynomial in $n$ ). Formally, given a $\operatorname{SIS}_{q, 2 m, \beta}$ instance $\hat{\mathbf{A}}=\left(\hat{\mathbf{A}}_{1} \| \hat{\mathbf{A}}_{2}\right) \in \mathbb{Z}_{q}^{n \times 2 m}, \mathcal{B}$ randomly chooses an integer $h^{*} \leftarrow_{R}[N]$. If $h^{*}=0, \mathcal{B}$ sets $\mathbf{A}=\hat{\mathbf{A}}$. Otherwise, $\mathcal{B}$ sets $\mathbf{A}=\left(h^{*} \hat{\mathbf{A}}_{1} \| \hat{\mathbf{A}}_{2}\right)$. Since $q$ is a prime and $N<q$ (i.e., $h^{*} \neq 0$ is invertible in $\mathbb{Z}_{q}$ ), we have that $\mathbf{A}$ is uniformly distributed over $\mathbb{Z}_{q}^{n \times 2 m}$. Then, $\mathcal{B}$ gives $\mathbf{A}=\left(\mathbf{A}_{1} \| \mathbf{A}_{2}\right)$ to $\mathcal{A}$, and obtains a solution $\left(\mathbf{x}=\left(\mathbf{x}_{1} ; \mathbf{x}_{2}\right), h\right) \in \mathbb{Z}^{2 m} \times[N]$ satisfying $\mathbf{A}_{1} \mathbf{x}_{1}+h \mathbf{A}_{2} \mathbf{x}_{2}=\mathbf{0}$. If $h^{*} \neq h, \mathcal{B}$ aborts. (Since $h^{*}$ is randomly chosen from $[N]$, the probability $\operatorname{Pr}\left[h^{*}=h\right]$ is at least $1 / N$.) Otherwise, $\mathcal{B}$ returns $\mathbf{y}=\left(\mathbf{x}_{1} ; \mathbf{0}\right)$ if $h^{*}=0$, else returns $\mathbf{y}=\mathbf{x}$. The first claim follows from the fact that $\mathbf{y} \neq 0,\|\mathbf{y}\| \leq \beta$ and $\hat{\mathbf{A}} \mathbf{y}=0$. Combining this with Proposition 2, the second claim follows.

\subsection{A Family of Hash Functions from Split-SIS Problems}

We define a new family of hash functions based on the split-SIS problem, which plays a key role in reducing the sizes of the group public key and the signature in our construction. Formally, for integers $n, m$, prime $q$, and polynomial $\beta=\beta(n) \geq$ $\omega(\sqrt{\log m}), N=N(n)<q$, we define $D_{m, \beta}=\left\{\mathbf{x} \leftarrow_{R} D_{\mathbb{Z}^{m}, \beta}:\|\mathbf{x}\| \leq \beta \sqrt{m}\right\}$, and a hash function family $\mathcal{H}_{n, m, q, \beta, N}=\left\{f_{\mathbf{A}}: D_{m, \beta, N} \rightarrow \mathbb{Z}_{q}^{n} \times D_{m, \beta}\right\}_{\mathbf{A} \in \mathbb{Z}_{q}^{n \times 2 m}}$, where $D_{m, \beta, N}:=D_{m, \beta} \times D_{m, \beta} \times[N]$. For index $\mathbf{A}=\left(\mathbf{A}_{1} \| \mathbf{A}_{2}\right) \in \mathbb{Z}_{q}^{n \times 2 m}$, and input $\left(\mathbf{x}_{1}, \mathbf{x}_{2}, h\right) \in D_{m, \beta, N}$, the hash value $f_{\mathbf{A}}\left(\mathbf{x}_{1}, \mathbf{x}_{2}, h\right):=\left(\mathbf{A}_{1} \mathbf{x}_{1}+h \mathbf{A}_{2} \mathbf{x}_{2}, \mathbf{x}_{2}\right) \in$ $\mathbb{Z}_{q}^{n} \times D_{m, \beta}$. In the following, we show three properties of $\mathcal{H}_{n, m, q, \beta, N}$, which are useful to construct zero-knowledge proofs for the function in $\mathcal{H}_{n, m, q, \beta, N}$.

Theorem 2 (One-Wayness). For parameters $m>2 n \log q, \beta=\beta(n)>2$. $\omega(\sqrt{\log m})$, prime $q=q(n)$, and polynomial $N=N(n)<q$, if the split$S I S_{q, m, \sqrt{5 m} \beta, N}$ problem is hard, then the family of hash functions $\mathcal{H}_{n, m, q, \beta, N}$ is one-way.

Proof. Assume that there is an algorithm $\mathcal{A}$ that breaks the one-wayness of $\mathcal{H}_{n, m, q, \beta, N}$, we construct an algorithm $\mathcal{B}$ that solves the split-SIS ${ }_{q, m, \sqrt{5 m} \beta, N}$ 
problem. Actually, given a split-SIS ${ }_{q, m, \sqrt{5 m} \beta, N}$ instance $\mathbf{A}=\left(\mathbf{A}_{1} \| \mathbf{A}_{2}\right) \in \mathbb{Z}_{q}^{n \times 2 m}$, $\mathcal{B}$ randomly chooses $\left(\mathbf{x}_{1}, \mathbf{x}_{2}\right) \in D_{\mathbb{Z}^{m}, \beta} \times D_{\mathbb{Z}^{m}, \beta}$ and $h \leftarrow_{R}[N]$, and computes $\mathbf{y}=f_{\mathbf{A}}\left(\mathbf{x}_{1}, \mathbf{x}_{2}, h\right)=\left(\mathbf{A}_{1} \mathbf{x}_{1}+h \mathbf{A}_{2} \mathbf{x}_{2}, \mathbf{x}_{2}\right)$. Then, it gives $(\mathbf{A}, \mathbf{y})$ to $\mathcal{A}$, and obtains $\left(\mathbf{x}_{1}^{\prime}, \mathbf{x}_{2}^{\prime}, h^{\prime}\right)$ satisfying $\left(\mathbf{A}_{1} \mathbf{x}_{1}^{\prime}+h^{\prime} \mathbf{A}_{2} \mathbf{x}_{2}^{\prime}, \mathbf{x}_{2}^{\prime}\right)=\mathbf{y}$. Finally, if $h \geq h^{\prime}, \mathcal{B}$ outputs $\left(\hat{\mathbf{x}}_{1}, \hat{\mathbf{x}}_{2}, \hat{h}\right)=\left(\mathbf{x}_{1}-\mathbf{x}_{1}^{\prime}, \mathbf{x}_{2}, h-h^{\prime}\right)$. Else, $\mathcal{B}$ outputs $\left(\hat{\mathbf{x}}_{1}, \hat{\mathbf{x}}_{2}, \hat{h}\right)=\left(\mathbf{x}_{1}^{\prime}-\mathbf{x}_{1}, \mathbf{x}_{2}, h^{\prime}-h\right)$.

It is easy to check that $\mathbf{A}_{1} \hat{\mathbf{x}}_{1}+\hat{h} \mathbf{A}_{2} \hat{\mathbf{x}}_{2}=\mathbf{0} \bmod q, \hat{h} \in[N]$, and $\left\|\left(\hat{\mathbf{x}}_{1} ; \hat{\mathbf{x}}_{2}\right)\right\| \leq$ $\sqrt{5 m} \beta$ with overwhelming probability by the standard tail inequality of the Gaussian distribution $D_{\mathbb{Z}^{m}, \beta}$. We finish this proof by showing that $\operatorname{Pr}\left[\hat{\mathbf{x}}_{1}=\mathbf{0}\right]$ is negligible in $n$. Note that $\mathcal{A}$ can only obtain the information about $\mathbf{x}_{1}$ from $\mathbf{A}_{1} \mathbf{x}_{1}$. By [36, Lem.5.2], this only leaks the distribution $\mathbf{t}+D_{\boldsymbol{\Lambda}_{q}^{\perp}\left(\mathbf{A}_{1}\right), \beta,-\mathbf{t}}$ for any $\mathbf{t}$ satisfying $\mathbf{A}_{1} \mathbf{t}=\mathbf{A}_{1} \mathbf{x}_{1}$. Namely, $\mathbf{x}_{1}$ should be uniformly distributed over $\mathbf{t}+D_{\mathbf{\Lambda}_{\frac{q}{q}}^{\perp}\left(\mathbf{A}_{1}\right), \beta,-\mathbf{t}}$ from the view of $\mathcal{A}$. Combining this with [56, Lem.2.16], we have $\operatorname{Pr}\left[\mathbf{x}_{1}=\mathbf{x}_{1}^{\prime}\right]$ is negligible in $n$. In other words, we have $\operatorname{Pr}[\hat{\mathbf{x}} \neq \mathbf{0}]=1-\operatorname{negl}(n)$, which completes the proof.

Since $f_{\mathbf{A}}\left(\mathbf{x}_{1}, \mathbf{0}, h\right)=f_{\mathbf{A}}\left(\mathbf{x}_{1}, \mathbf{0}, 0\right)$ holds for all $h \in[N]$, the function $\mathcal{H}_{n, m, q, \beta, N}$ with domain $D_{m, \beta, N}:=D_{m, \beta} \times D_{m, \beta} \times[N]$ are not collision-resistant. However, if we slightly restrict the domain of $\mathcal{H}_{n, m, q, \beta, N}$ to exclude the above trivial case, we can prove that the family of $\mathcal{H}_{n, m, q, \beta, N}$ is collision-resistant. Formally, we slightly restrict the domain of $\mathcal{H}_{n, m, q, \beta, N}$ to be $D_{m, \beta, N}^{\prime}=\left\{\left(\mathbf{x}_{1}, \mathbf{x}_{2}, h\right) \in D_{m, \beta, N}\right.$ : $\left.\mathbf{x}_{2} \neq \mathbf{0}\right\}$.

Theorem 3 (Collision-Resistance). For parameter $m=m(n), \beta=\beta(n)$, prime $q=q(n)$, and polynomial $N=N(n)<q$, if the split-SIS ${ }_{q, m, \sqrt{5 m} \beta, N}$ problem is hard, then the family of hash functions $\mathcal{H}_{n, m, q, \beta, N}$ with domain $D_{m, \beta, N}^{\prime}$ is collision-resistant.

Proof. Assume there is a PPT algorithm $\mathcal{A}$ that can find collisions of $\mathcal{H}_{n, m, q, \beta, N}$ with non-negligible probability $\epsilon$, we construct an algorithm $\mathcal{B}$ solving splitSIS $_{q, m, \sqrt{5 m} \beta, N}$ with the same probability. Concretely, after obtaining a splitSIS $_{q, m, \sqrt{5 m} \beta, N}$ instance $\mathbf{A}=\left(\mathbf{A}_{1} \| \mathbf{A}_{2}\right), \mathcal{B}$ directly gives $\mathbf{A}$ to $\mathcal{A}$, and obtains a pair of collisions $\left(\mathbf{x}_{1}, \mathbf{x}_{2}, h\right) \in D_{m, \beta, N}^{\prime}$ and $\left(\mathbf{x}_{1}^{\prime}, \mathbf{x}_{2}^{\prime}, h^{\prime}\right) \in D_{m, \beta, N}^{\prime}$ satisfying $\left(\mathbf{x}_{1}, \mathbf{x}_{2}, h\right) \neq\left(\mathbf{x}_{1}^{\prime}, \mathbf{x}_{2}^{\prime}, h^{\prime}\right)$ and $f_{\mathbf{A}}\left(\mathbf{x}_{1}, \mathbf{x}_{2}, h\right)=f_{\mathbf{A}}\left(\mathbf{x}_{1}^{\prime}, \mathbf{x}_{2}^{\prime}, h^{\prime}\right)$. Note that in this case, we must have $\mathbf{x}_{2}=\mathbf{x}_{2}^{\prime} \neq \mathbf{0}$. If $h \geq h^{\prime}, \mathcal{B}$ returns $\left(\hat{\mathbf{x}}_{1}, \hat{\mathbf{x}}_{2}, \hat{h}\right)=\left(\mathbf{x}_{1}-\right.$ $\left.\mathbf{x}_{1}^{\prime}, \mathbf{x}_{2}, h-h^{\prime}\right)$, else it returns $\left(\hat{\mathbf{x}}_{1}, \hat{\mathbf{x}}_{2}, \hat{h}\right)=\left(\mathbf{x}_{1}^{\prime}-\mathbf{x}_{1}, \mathbf{x}_{2}, h^{\prime}-h\right)$. By the assumption that $\left(\mathbf{x}_{1}, \mathbf{x}_{2}, h\right) \neq\left(\mathbf{x}_{1}^{\prime}, \mathbf{x}_{2}^{\prime}, h^{\prime}\right)$, the inequality $\left(\hat{\mathbf{x}}_{1}, \hat{h}\right) \neq \mathbf{0}$ holds in both cases, i.e., we always have $\hat{\mathbf{x}}_{1} \neq \mathbf{0}$ or $\hat{h} \hat{\mathbf{x}}_{2} \neq \mathbf{0}$. The claim follows from the fact that $\left\|\left(\hat{\mathbf{x}}_{1} ; \hat{\mathbf{x}}_{2}\right)\right\| \leq \sqrt{5 m} \beta$ and $\hat{h} \in[N]$.

Finally, we show that the family of hash functions $\mathcal{H}_{n, m, q, \beta, N}$ statistically hides its third input.

Theorem 4. Let parameter $m>2 n \log q, \beta=\beta(n)>\omega(\sqrt{\log m})$, prime $q=$ $q(n)$, and polynomial $N=N(n)$. Then, for a randomly chosen $\mathbf{A}=\left(\mathbf{A}_{1} \| \mathbf{A}_{2}\right) \in$ $\mathbb{Z}_{q}^{n \times 2 m}$, and arbitrarily $\mathbf{x}_{2}$ with norm $\left\|\mathbf{x}_{2}\right\| \leq \beta \sqrt{m}$, the statistical distance between the following two distributions:

$$
\left\{\left(\mathbf{A}, f_{\mathbf{A}}\left(\mathbf{x}_{1}, \mathbf{x}_{2}, h\right), h\right): \mathbf{x}_{1} \leftarrow_{R} D_{m, \beta}, h \leftarrow_{R}[N]\right\}
$$


and

$$
\left\{\left(\mathbf{A},\left(\mathbf{u}, \mathbf{x}_{2}\right), h\right): \mathbf{u} \leftarrow_{R} \mathbb{Z}_{q}^{n}, h \leftarrow_{R}[N]\right\}
$$

is negligible in $n$.

Proof. Since the second output of $f_{\mathbf{A}}\left(\mathbf{x}_{1}, \mathbf{x}_{2}, h\right)$ (i.e., $\left.\mathbf{x}_{2}\right)$ is independent from the choices of $h$, we only have to show that, for arbitrarily $\mathbf{x}_{2}$ and $h$, the distribution $\left\{\mathbf{A}_{1} \mathbf{x}_{1}+h \mathbf{A}_{2} \mathbf{x}_{2}: \mathbf{x}_{1} \leftarrow_{R} D_{m, \beta}\right\}$ is statistically close to uniform over $\mathbb{Z}_{q}^{n}$. Actually, using the fact that $\beta \geq \omega(\sqrt{\log m})$ together with Lemma 3 , we have that the distribution of $\mathbf{A}_{1} \mathbf{x}_{1}$ is statistically close to uniform over $\mathbb{Z}_{q}^{n}$ when $\mathbf{x}_{1} \leftarrow_{R} D_{\mathbb{Z}^{m}, \beta}$. The claim of this theorem follows from the fact that the statistical distance between $D_{\mathbb{Z}^{m}, \beta}$ and $D_{m, \beta}$ is negligible, and that the distribution $\left\{\mathbf{u}+h \mathbf{A}_{2} \mathbf{x}_{2}: \mathbf{u} \leftarrow_{R} \mathbb{Z}_{q}^{n}\right\}$ is exactly the uniform distribution over $\mathbb{Z}_{q}^{n}$ for arbitrary $\mathbf{x}_{2} \in D_{m, \beta}, h \in[N]$.

\subsection{Zero-Knowledge Proof of Knowledge for the Hash Functions}

In this subsection, we present a proof of knowledge protocol for the family of hash functions $\mathcal{H}_{n, m, q, \beta, N}$. Concretely, given a matrix $\mathbf{A}=\left(\mathbf{A}_{1} \| \mathbf{A}_{2}\right)$, a vector $\mathbf{y}=\left(\mathbf{y}_{1}, \mathbf{y}_{2}\right) \in \mathbb{Z}_{q}^{n} \times \mathbb{Z}^{m}$ with $0<\left\|\mathbf{y}_{2}\right\| \leq \beta \sqrt{m}$, the prover can generate a proof of knowledge of $\mathbf{x}=\left(\mathbf{x}_{1}, \mathbf{x}_{2}, h\right) \in \mathbb{Z}^{2 m+1}$ satisfying $\left\|\mathbf{x}_{1}\right\| \leq \beta \sqrt{m}, h \in[N]$ and $f_{\mathbf{A}}\left(\mathbf{x}_{1}, \mathbf{x}_{2}, h\right)=\left(\mathbf{A} \mathbf{x}_{1}+h \mathbf{A}_{2} \mathbf{x}_{2}, \mathbf{x}_{2}\right)=\mathbf{y}$. Since $\mathbf{x}_{2}$ must be equal to $\mathbf{y}_{2}$, the protocol is actually a proof of knowledge for the relation

$$
\begin{aligned}
R_{\text {split-SIS }}=\left\{\left(\mathbf{A}, \mathbf{y}, \beta, N ; \mathbf{x}_{1}, h\right)\right. & \in \mathbb{Z}_{q}^{n \times 2 m} \times\left(\mathbb{Z}_{q}^{n} \times \mathbb{Z}^{m}\right) \times \mathbb{R} \times \mathbb{Z} \times \mathbb{Z}^{m} \times \mathbb{Z}: \\
& \left.\mathbf{A}_{1} \mathbf{x}_{1}+h \mathbf{A}_{2} \mathbf{y}_{2}=\mathbf{y}_{1},\left\|\mathbf{x}_{1}\right\| \leq \beta \sqrt{m} \text { and } h \in[N]\right\} .
\end{aligned}
$$

Intuitively, we can adapt a variant of the protocols for ISIS relations in $[43,49,50]$ for our purpose, since one can rewrite $\mathbf{y}_{1}=\mathbf{A}_{1} \mathbf{x}_{1}+h \mathbf{A}_{2} \mathbf{y}_{2}=$ $\left(\mathbf{A}_{1} \| \mathbf{A}_{2} \mathbf{y}_{2}\right)\left(\mathbf{x}_{1} ; h\right)$. However, this may not work when $N \gg \beta$. Since the basic idea of $[43,49,50]$ is to use randomness from a "large width" distribution (compared to the distribution of the witness) to hide the distribution of the witness, the width of the randomness distribution should be sufficiently larger than $N$ in our case, which might lead to a proof without soundness guarantee.

Fortunately, we can borrow the "bit-decomposition" technique from [22-24] to deal with large $N$. The idea is to decompose $h \in[N]$ into a vector of small elements, and then prove the existence of such a vector for $h$. Formally, for any $h \in[N]$, we compute the representation of $h$ in base $\bar{\beta}=\lfloor\beta\rfloor$, namely, a $\ell$-dimension vector $\mathbf{v}_{h}=\left(v_{0}, \ldots, v_{\ell-1}\right) \in \mathbb{Z}^{\ell}$ such that $0 \leq v_{i} \leq \beta-1$ and $h=\sum_{i=0}^{\ell-1} v_{i} \bar{\beta}^{i}$, where $\ell=\left\lceil\log _{\bar{\beta}} N\right\rceil$. Denote $\mathbf{b}=\mathbf{A}_{2} \mathbf{y}_{2}$, compute $\mathbf{D}=$ $\left(\mathbf{b}, \bar{\beta} \mathbf{b}, \ldots, \bar{\beta}^{\ell-1} \mathbf{b}\right) \in \mathbb{Z}_{q}^{n \times \ell}$. It is easy to check that for any vector $\mathbf{e} \in \mathbb{Z}^{\ell}$, there exists a $h^{\prime} \in \mathbb{Z}_{q}$ such that $\mathbf{D e}=h^{\prime} \mathbf{b} \bmod q .\left(h^{\prime} \in \mathbb{Z}_{q}\right.$ is unique if $\mathbf{b} \neq \mathbf{0}$.) In particular, we have that $\mathbf{y}_{1}=\hat{\mathbf{A}} \hat{\mathbf{x}}$, where $\hat{\mathbf{A}}=\left(\mathbf{A}_{1} \| \mathbf{D}\right) \in \mathbb{Z}_{q}^{n \times(m+\ell)}$, $\hat{\mathbf{x}}=\left(\mathbf{x}_{1} ; \mathbf{v}_{h}\right) \in \mathbb{Z}^{m+\ell}$ and $\|\hat{\mathbf{x}}\| \leq \beta \sqrt{m+\ell}$. Since $\bar{\beta}>2$ and $N$ is a polynomial in $n$, we have $\ell \ll m$ and $\|\hat{\mathbf{x}}\|<\eta=\beta \sqrt{2 m}$.

We first present a $\Sigma$-protocol for the function family $\mathcal{H}_{n, m, q, \beta, N}$, which repeats a basic protocol with single-bit challenge $t=\omega(\log n)$ times in parallel. As 
in $[43,50]$, the basic protocol makes use of the rejection sampling technique to achieve zero-knowledge. Formally, let $\gamma=\eta \cdot m^{1.5}$, denote $\zeta(\mathbf{z}, \mathbf{y})=1-$ $\min \left(\frac{D_{\mathbb{Z}^{m+\ell, \gamma}}(\mathbf{z})}{M_{l} \cdot D_{\mathbb{Z}^{m+\ell, y, \gamma}}(\mathbf{z})}, 1\right)$, where $\mathbf{y}, \mathbf{z} \in \mathbb{Z}^{m+\ell}$, and the constant $M_{l} \leq 1+O\left(\frac{1}{m}\right)$ is set according to Lemma 4.5 in [50], the protocol is depicted in Fig 2.

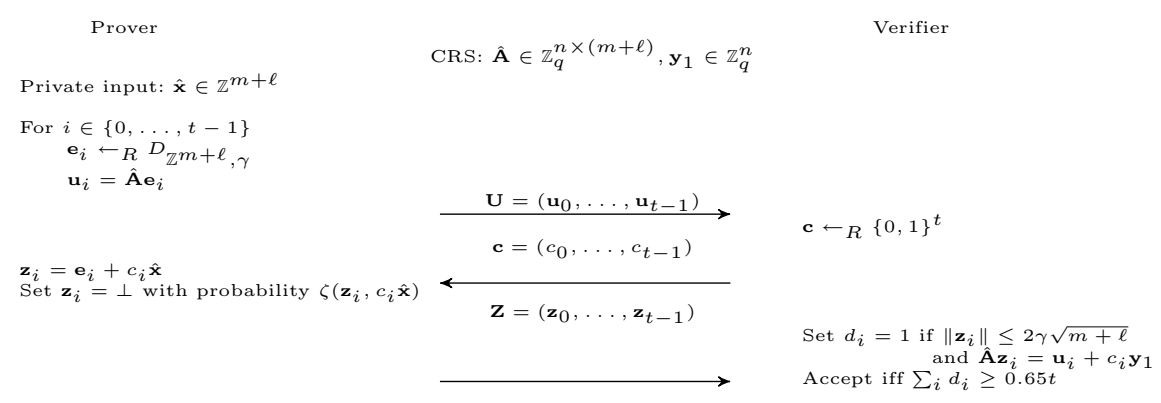

Fig. 2. $\Sigma$-protocol for $R_{\text {split-SIS }}$

By [50, Th.4.6], we have $\operatorname{Pr}\left[\mathbf{z}_{i} \neq \perp\right] \approx \frac{1}{M_{l}}=1-O\left(\frac{1}{m}\right)$ for each $i \in\{0, \ldots, t-$ 1\}. In addition, $\operatorname{Pr}\left[\left\|\mathbf{z}_{i}\right\| \leq 2 \gamma \sqrt{m+\ell} \mid \mathbf{z}_{i} \neq \perp\right]=1-\operatorname{negl}(m)$ by [50, Lem.4.4]. A simple calculation shows that the completeness error of the protocol is at most $2^{-\Omega(t)}$ (when $m$ is sufficiently large, e.g., $m>100$ ). Besides, the protocol has the property of special Honest-Verifier Zero Knowledge (HVZK). Namely, given a challenge $c_{i}$, there exists a simulator $\mathcal{S}$ that outputs a distribution $\left(\mathbf{u}_{i}, c_{i}, \mathbf{z}_{i}\right)$ statistically close to the real transcript distribution. Concretely, $\mathcal{S}$ first chooses $\mathbf{z}_{i} \leftarrow_{R} D_{\mathbb{Z}^{m+\ell}, \gamma}$, and computes $\mathbf{u}_{i}=\hat{\mathbf{A}} \mathbf{z}_{i}-c_{i} \mathbf{y}_{1} \bmod q$. Then, it sets $\mathbf{z}_{i}=\perp$ with probability $1-\frac{1}{M_{l}}$, and outputs $\left(\mathbf{u}_{i}, c_{i}, \mathbf{z}_{i}\right)$. By Theorem 4 , the term $\hat{\mathbf{A}} \mathbf{z}_{i}(\bmod q)$ is statistically close to uniform over $\mathbb{Z}_{q}^{n}$, thus the distribution of $\mathbf{u}_{i}$ is statistically close to that in the real proof. Moreover, by [50, Th.4.6], the distribution of $\mathbf{z}_{i}$ is also statistically close to that in the real transcripts.

Finally, since the binary challenges (i.e., c ) are used, the above protocol has the property of special soundness. Actually, given two transcripts $(\mathbf{U}, \mathbf{c}, \mathbf{Z})$ and $\left(\mathbf{U}, \mathbf{c}^{\prime}, \mathbf{Z}^{\prime}\right)$ with distinct challenges $\mathbf{c} \neq \mathbf{c}^{\prime}$, one can extract a "weak" witness $\mathbf{x}^{\prime}=\mathbf{z}_{i}-\mathbf{z}_{i}^{\prime}$ for some $i$ satisfying $\hat{\mathbf{A}} \mathbf{x}^{\prime}=\mathbf{y}_{1}$ and $\left\|\mathbf{x}^{\prime}\right\| \leq 4 \gamma \sqrt{2 m}$.

Applying the "Fiat-Shamir Heuristic" [34] in a standard way, one can obtain an NIZKPoK by computing $\mathbf{c}=H(\rho, \mathbf{U})$, where $H:\{0,1\}^{*} \rightarrow\{0,1\}^{t}$ is modeled as a random oracle, and $\rho$ represents all the other auxiliary inputs, e.g., a specified message $M$ to be signed. Finally, due to the nice property of $\Sigma$-protocol, one can easily combine the protocol to prove EQ-relation, OR-relation, and ANDrelation, we omit the details. 


\section{A Simple and Efficient Group Signature from Lattices}

In this section, we present our CPA-anonymous lattice-based group signature, which can be easily extended to support CCA anonymity by replacing the underlying encryption with a CCA one. We defer the full-anonymous scheme to the full version.

\subsection{Our Construction}

Assume that the security parameter is $n$, and $\delta$ is a real such that $n^{1+\delta}>$ $\lceil(n+1) \log q+n\rceil$, all other parameters $m, s, \alpha, \beta, \eta, p, q$ are determined as follows:

$$
\begin{aligned}
& m=6 n^{1+\delta} \\
& s=m \cdot \omega(\log m) \\
& \beta=s \sqrt{2 m} \cdot \omega(\sqrt{\log 2 m})=m^{1.5} \cdot \omega\left(\log ^{1.5} m\right) \\
& p=m^{2.5} \beta=m^{4} \cdot \omega\left(\log ^{1.5} m\right) \\
& q=m^{2} \cdot \max \left(p m^{2.5} \cdot \omega(\log m), 4 N\right)=m^{2.5} \max \left(m^{6} \cdot \omega\left(\log ^{2.5} m\right), 4 N\right) \\
& \alpha=2 \sqrt{m} / q \\
& \eta=\max (\beta, \alpha q) \sqrt{m}=m^{2} \cdot \omega\left(\log ^{1.5} m\right)
\end{aligned}
$$

Now, we present our group signature $\mathcal{G S}=($ KeyGen, Sign, Verify, Open $)$ :

- KeyGen $\left(1^{n}, 1^{N}\right)$ : Take the security parameter $n$ and the maximum number $N$ of group members as inputs, set an integer $m \in \mathbb{Z}$, primes $p, q \in \mathbb{Z}$, and $s, \alpha, \beta, \eta \in \mathbb{R}$ as above, and choose a hash function $H:\{0,1\}^{*} \rightarrow\{0,1\}^{t}$ (modeled as random oracle) for the NIZKPoK proof, where $t=\omega(\log n)$. Then, the algorithm proceeds as follows:

1. Compute $\left(\mathbf{A}_{1}, \mathbf{T}_{\mathbf{A}_{1}}\right) \leftarrow \operatorname{TrapGen}(n, m, q)$, and randomly choose $\mathbf{A}_{2,1}$, $\mathbf{A}_{2,2} \leftarrow{ }_{R} \mathbb{Z}_{q}^{n \times m}$

2. Compute $\left(\mathbf{B}, \mathbf{T}_{\mathbf{B}}\right) \leftarrow \operatorname{SuperSamp}\left(n, m, q, \mathbf{A}_{1}, \mathbf{0}\right)$.

3. For $j=1, \ldots, N$, define $\overline{\mathbf{A}}_{j}=\left(\mathbf{A}_{1} \| \mathbf{A}_{2,1}+j \mathbf{A}_{2,2}\right)$, extract a basis $\mathbf{T}_{\overline{\mathbf{A}}_{j}} \leftarrow$ $\operatorname{ExtRndBasis}\left(\overline{\mathbf{A}}_{j}, \mathbf{T}_{\mathbf{A}_{1}}, s\right)$ such that $\left\|\widetilde{\mathbf{T}}_{\overline{\mathbf{A}}_{j}}\right\| \leq s \sqrt{2 m}$.

4. Define the group public key $g p k=\left\{\mathbf{A}_{1}, \mathbf{A}_{2,1}, \mathbf{A}_{2,2}, \mathbf{B}\right\}$, the group manager secret key gmsk $=\mathbf{T}_{\mathbf{B}}$, and the group member's secret keys gsk $=$ $\left\{g s k_{j}=\mathbf{T}_{\overline{\mathbf{A}}_{j}}\right\}_{j \in\{1, \ldots, N\}}$

- Sign $\left(g p k, g s k_{j}, M\right)$ : Take the group public key $g p k=\left\{\mathbf{A}_{1}, \mathbf{A}_{2,1}, \mathbf{A}_{2,2}, \mathbf{B}\right\}$, the $j$-th user's secret key $g s k_{j}=\mathbf{T}_{\overline{\mathbf{A}}_{j}}$, and a message $M \in\{0,1\}^{*}$ as inputs, proceed as follows:

1. Compute $\left(\mathbf{x}_{1}, \mathbf{x}_{2}\right) \leftarrow$ SamplePre $\left(\overline{\mathbf{A}}_{j}, \mathbf{T}_{\overline{\mathbf{A}}_{j}}, \beta, \mathbf{0}\right)$, where $\mathbf{x}_{1}, \mathbf{x}_{2} \in D_{\mathbb{Z}^{m}, \beta}$.

2. Choose $\mathbf{s} \leftarrow_{R} \mathbb{Z}_{q}^{n}, \mathbf{e} \leftarrow{ }_{R} \chi_{\alpha}^{m}$, and compute

$$
\mathbf{c}=\mathbf{B}^{T} \mathbf{s}+p \mathbf{e}+\mathbf{x}_{1}
$$

3. Generate a NIZKPoK proof $\pi_{1}$ of $\left(\mathbf{s}, \mathbf{e}, \mathbf{x}_{1}\right)$ such that $\left(\mathbf{B}, \mathbf{c}, \eta ; \mathbf{s}, \mathbf{e}, \mathbf{x}_{1}\right) \in$ $R_{\text {eLWE }}$. 
4. Let $\bar{\beta}:=\lfloor\beta\rfloor$ and $\ell=\left\lceil\log _{\bar{\beta}} N\right\rceil$, define $\mathbf{b}=\mathbf{A}_{2,2} \mathbf{x}_{2}$, and $\mathbf{D}=$ $\left(\mathbf{b}, \bar{\beta} \mathbf{b}, \ldots, \bar{\beta}^{\ell-1} \mathbf{b}\right) \in \mathbb{Z}_{q}^{n \times \ell}$. Generate a NIZKPoK $\pi_{2}$ of $\mathbf{x}_{1}, \mathbf{e}$, and $\mathbf{v}_{j}=\left(v_{0}, \ldots, v_{\ell-1}\right) \in \mathbb{Z}_{\bar{\beta}}^{\ell}$ of $j \in[N]$ such that,

$$
\begin{gathered}
\mathbf{A}_{1} \mathbf{c}+\mathbf{A}_{2,1} \mathbf{x}_{2}=\left(p \mathbf{A}_{1}\right) \mathbf{e}-\mathbf{D} \mathbf{v}_{j}, \text { and } \\
\mathbf{A}_{1} \mathbf{c}=\left(p \mathbf{A}_{1}\right) \mathbf{e}+\mathbf{A}_{1} \mathbf{x}_{1}
\end{gathered}
$$

where the challenge is computed by $H\left(\mathbf{c}, \mathbf{x}_{2}, \pi_{1}, M\right.$, Com $)$, and Com is the commitment message for the NIZKPoK proof of $\pi_{2}$. (Note that the proof, i.e., $\pi_{2}$, is actually a standard composition of our protocol in Section 4.2 and the protocol for $R_{\mathrm{ISIS}}[43,50]$ according to the nice property of the underlying $\Sigma$-protocol. More discussions are given in the full version.)

5. Output the signature $\sigma=\left(\mathbf{c}, \mathbf{x}_{2}, \pi_{1}, \pi_{2}\right)$.

- Verify $(g p k, M, \sigma)$ : Parse $\sigma=\left(\mathbf{c}, \mathbf{x}_{2}, \pi_{1}, \pi_{2}\right)$, return 1 if $\left\|\mathbf{x}_{2}\right\| \leq \beta \sqrt{m}$, $\mathbf{A}_{2,2} \mathbf{x}_{2} \neq \mathbf{0}$, and the proofs $\pi_{1}, \pi_{2}$ are valid, else return 0 .

- Open $(g p k, g m s k, M, \sigma)$ : Parse $g p k=\left\{\mathbf{A}_{1}, \mathbf{A}_{2,1}, \mathbf{A}_{2,2}, \mathbf{B}\right\}$ and $g m s k=\mathbf{T}_{\mathbf{B}}$, compute $\mathbf{x}_{1}$ by decrypting $\mathbf{c}$ using $\mathbf{T}_{\mathbf{B}}$. Then, compute $\mathbf{y}_{0}=\mathbf{A}_{2,2} \mathbf{x}_{2}$ and $\mathbf{y}_{1}=-\mathbf{A}_{1} \mathbf{x}_{1}-\mathbf{A}_{2,1} \mathbf{x}_{2}$. If $\mathbf{y}_{0} \neq \mathbf{0}$ and there is a $j \in \mathbb{Z}_{q}^{*}$ such that $\mathbf{y}_{1}=j \cdot \mathbf{y}_{0}$ $\bmod q$, output $j$, else output $\perp$.

Remark 1. One can decrypt $\mathbf{c}$ by first computing $\mathbf{T}_{\mathbf{B}}^{T} \cdot \mathbf{c}=\mathbf{T}_{\mathbf{B}}^{T}\left(p \mathbf{e}+\mathbf{x}_{1}\right) \bmod q$. If $\left\|\mathbf{T}_{\mathbf{B}}^{T}\left(p \mathbf{e}+\mathbf{x}_{1}\right)\right\|_{\infty}<q / 2$, one can expect that $\mathbf{T}_{\mathbf{B}}^{T}\left(p \mathbf{e}+\mathbf{x}_{1}\right)=\left(\mathbf{T}_{\mathbf{B}}^{T} \mathbf{c} \bmod q\right)$ holds over $\mathbb{Z}$. Thus, $\hat{\mathbf{x}}=\left(p \mathbf{e}+\mathbf{x}_{1}\right)$ can be solved by using Gaussian elimination over $\mathbb{Z}$ since $\mathbf{T}_{\mathbf{B}} \in \mathbb{Z}^{m \times m}$ is full-rank. Finally, $\mathbf{x}_{1}=\hat{\mathbf{x}} \bmod p$ can be successfully recovered if $\left\|\mathbf{x}_{1}\right\|_{\infty}<p / 2$.

For the correctness of our group signature scheme, we have the following theorem.

Theorem 5. Assume $n$ is the security parameter, and all other parameters $m, s, \alpha, \beta, \eta, p, q$ are functions of $n$ defined as in (1), where $p, q$ are primes. Then, the group signature $\mathcal{G S}$ is correct, and the group public key and the signature have bit-length $4 n m \log q$ and $O(t m \log q)$, respectively, where $t=\omega(\log n)$.

Proof. Since we set $m=6 n^{1+\delta}>\lceil 6 n \log q+n\rceil$, the two algorithms TrapGen and SuperSamp can work correctly with overwhelming probability. In particular, we have $\left\|\tilde{\mathbf{T}}_{\mathbf{A}_{1}}\right\| \leq O(\sqrt{m})$, and $\left\|\mathbf{T}_{\mathbf{B}}\right\| \leq m^{1.5} \cdot O(\sqrt{\log m})$ by Proposition 3 and Proposition 6. By Proposition 4, we have $\left\|\widetilde{\mathbf{T}}_{\overline{\mathbf{A}}_{j}}\right\| \leq s \sqrt{2 m}$ for all $i \in\{1, \ldots, N\}$ with overwhelming probability. Since the group public key only contains four matrices over $\mathbb{Z}_{q}^{n \times m}$, it has bit-size $4 n m \log q=O(n m \log q)$.

For the Sign algorithm, since $\beta=s \sqrt{2 m} \cdot \omega(\sqrt{\log 2 m}) \geq\left\|\widetilde{\mathbf{T}}_{\overline{\mathbf{A}}_{j}}\right\| \cdot \omega(\sqrt{\log 2 m})$, we have $\mathbf{x}_{1}, \mathbf{x}_{2} \backsim D_{\mathbb{Z}^{m}, \beta}$ by the correctness of the SamplePre algorithm in [36], and $\left\|\mathbf{x}_{i}\right\| \leq \beta \sqrt{m}$ with overwhelming probability. In addition, since $\mathbf{e}$ is chosen from $\chi_{\alpha}$, we have $\|\mathbf{e}\| \leq \alpha q \sqrt{m}$ with overwhelming probability. By the choices of $\eta=\max (\beta, \alpha q) \sqrt{m}$, the algorithm can successfully generate the proofs $\pi_{1}$ and $\pi_{2}$. For the bit-length of the signature $\sigma=\left(\mathbf{c}, \mathbf{x}_{2}, \pi_{1}, \pi_{2}\right)$, we know that 
both the bit-length of $\mathbf{c}$ and $\mathbf{x}_{2}$ are at most $m \log q$. In addition, if we set the repetition parameter $t=\omega(\log n)$ for the proof $\pi_{1}$ and $\pi_{2}$, the bit-length of $\pi_{1}$ and $\pi_{2}$ are at most $(3 m-n) t \log q$ and $(2 m+2 n+\ell) t \log q$, respectively. Thus, the total bit-length of the signature $\sigma$ is less than $2 m \log q+(5 m+n+\ell) t \log q=$ $O(t(m+\log N) \log q)=O(t m \log q)$ since $\ell=\left\lceil\log _{\bar{\beta}} N\right\rceil \ll n$ and $m=O(n \log q)$.

Note that $\mathbf{x}_{2} \backsim D_{\mathbb{Z}^{m}, \beta},{ }^{1}$ therefore $\operatorname{Pr}\left[\mathbf{A}_{2,2} \mathbf{x}_{2}=\mathbf{0}\right] \leq O\left(q^{-n}\right)$ by Lemma 3. Moreover, by the completeness of $\pi_{1}$ and $\pi_{2}$, the algorithm Verify will work correctly with overwhelming probability. As for the Open algorithm, we only have to show that we can correctly decrypt $\mathbf{x}_{1}$ from $\mathbf{c}$ by using $\mathbf{T}_{\mathbf{B}}$. Since $\mathbf{T}_{\mathbf{B}}^{T} \cdot \mathbf{c}=$ $\mathbf{T}_{\mathbf{B}}^{T}\left(p \mathbf{e}+\mathbf{x}_{1}\right) \bmod q$ holds, one can expect that $\mathbf{T}_{\mathbf{B}}^{T}\left(p \mathbf{e}+\mathbf{x}_{1}\right)=\left(\mathbf{T}_{\mathbf{B}}^{T} \mathbf{c} \bmod q\right)$ holds over $\mathbb{Z}$ if $\left\|\mathbf{T}_{\mathbf{B}}^{T}\left(p \mathbf{e}+\mathbf{x}_{1}\right)\right\|_{\infty}<q / 2$. Thus, one can solve $\hat{\mathbf{x}}=\left(p \mathbf{e}+\mathbf{x}_{1}\right)$ by Gaussian elimination over $\mathbb{Z}$ since $\mathbf{T}_{\mathbf{B}} \in \mathbb{Z}^{m \times m}$ is full-rank. Moreover, by the choices of $p$ and $\beta$, we have $\left\|\mathbf{x}_{1}\right\|_{\infty} \leq\left\|\mathbf{x}_{1}\right\|<p$, therefore $\mathbf{x}_{1}$ can be recovered by computing $\hat{\mathbf{x}} \bmod p$. We finish this proof by showing that $\left\|\mathbf{T}_{\mathbf{B}}^{T}\left(p \mathbf{e}+\mathbf{x}_{1}\right)\right\|_{\infty}<$ $q / 2$. Actually, by Lemma 2 and Lemma 1, we have $\left\|p \mathbf{e}+\mathbf{x}_{1}\right\| \leq 3 m^{6} \cdot \omega\left(\log ^{3} m\right)$. By Proposition 6 , we have $\left\|\mathbf{T}_{\mathbf{B}}\right\| \leq m^{1.5} \cdot \omega(\sqrt{\log m})$. It is easy to check that $\left\|\mathbf{T}_{\mathbf{B}}^{T}\left(p \mathbf{e}+\mathbf{x}_{1}\right)\right\|_{\infty} \leq 3 m^{8} \cdot \omega\left(\log ^{3.5} m\right) \ll q$, which satisfies the requirement.

\subsection{The Security}

For security, namely CPA-anonymity and full traceability, we have the following two theorems.

Theorem 6. CPA-Anonymity] Under the LWE assumption, our group signature $\mathcal{G S}$ is $C P A$-anonymous in the random oracle model.

Proof. We prove Theorem 6 via a sequence of games.

In game $G_{0}$, the challenger honestly generates the group public key $g p k=$ $\left\{\mathbf{A}_{1}, \mathbf{A}_{2,1}, \mathbf{A}_{2,2}, \mathbf{B}\right\}$, the group manager secret key gmsk $=\mathbf{T}_{\mathbf{B}}$, and the group member's secret key gsk $=\left\{g s k_{j}=\mathbf{T}_{\overline{\mathbf{A}}_{j}}\right\}_{j \in\{1, \ldots, N\}}$ by running the KeyGen algorithm. Then, it gives (gpk, gsk) to the adversary $\mathcal{A}$, and obtains a message $M$, and two user indexes $i_{0}, i_{1} \in\{1, \ldots, N\}$. Finally, the challenger randomly chooses a bit $b \leftarrow_{R}\{0,1\}$, computes $\sigma^{*}=\left(\mathbf{c}^{*}, \mathbf{x}_{2}^{*}, \pi_{1}^{*}, \pi_{2}^{*}\right) \leftarrow \operatorname{Sign}\left(g p k, g s k_{i_{b}}, M\right)$, and returns $\sigma^{*}$ to $\mathcal{A}$.

In Game $G_{1}$, the challenger behaves almost the same as in $G_{0}$, except that it uses the NIZKPoK simulators (by appropriately programming the random oracle) to generate $\pi_{1}^{*}, \pi_{2}^{*}$. By the property of the NIZKPoKs, $G_{1}$ is computationally indistinguishable from $G_{0}$.

In Game $G_{2}$, the challenger behaves almost the same as in $G_{1}$, except that it first chooses $\mathbf{x}_{2}^{*}$ from $D_{\mathbb{Z}^{m}, \beta}$, and then uses $\mathbf{T}_{\mathbf{A}_{1}}$ to extract $\mathbf{x}_{1}^{*}$ such that

${ }^{1}$ As noted by Cash et al. [31], the output distribution of the SamplePre algorithm in [36] is statistically close to the distribution $\left(\mathbf{x}_{1}, \mathbf{x}_{2}\right)$ that samples as follows: randomly choose $\mathbf{x}_{2} \leftarrow_{R} D_{\mathbb{Z}^{m}, s}$, and then compute $\mathbf{x}_{1}$ using $\mathbf{T}_{\mathbf{A}_{1}}$ to satisfy the condition $\mathbf{A}_{1} \mathbf{x}_{1}+\left(\mathbf{A}_{2,1}+j \mathbf{A}_{2,2}\right) \mathbf{x}_{2}=\mathbf{0}$. We note that this is also the reason why we do not encrypt $\mathbf{x}_{2}$ as in [43], since it leaks little information about $j$ without $\mathbf{x}_{1}$. 
$\overline{\mathbf{A}}_{i_{b}}\left(\mathbf{x}_{1}^{*} ; \mathbf{x}_{2}^{*}\right)=\mathbf{0}$. By the property of the SamplePre algorithm from [31,36], Game $G_{2}$ is statistically close to Game $G_{1}$

In Game $G_{3}$, the challenger behaves almost the same as in $G_{2}$, except that it computes $\mathbf{c}^{*}=\mathbf{u}+\mathbf{x}_{1}^{*}$ with a randomly chosen $\mathbf{u} \leftarrow_{R} \mathbb{Z}_{q}^{m}$.

Lemma 4. Under the LWE assumption, Game $G_{3}$ is computationally indistinguishable from Game $G_{2}$.

Proof. Assume there is an algorithm $\mathcal{A}$ which distinguishes $G_{2}$ from $G_{3}$ with non-negligible probability. Then, there is an algorithm $\mathcal{B}$ that breaks the LWE assumption. Formally, given a LWE tuple $(\hat{\mathbf{B}}, \hat{\mathbf{u}}) \in \mathbb{Z}_{q}^{n \times m} \times \mathbb{Z}_{q}^{m}, \mathcal{B}$ sets $\mathbf{B}=p \hat{\mathbf{B}}$, and computes $\left(\mathbf{A}_{1}, \mathbf{T}_{\mathbf{A}_{1}}\right) \leftarrow \operatorname{SuperSamp}(n, m, q, \mathbf{B}, \mathbf{0})$. Then, it chooses $\mathbf{A}_{2,1}, \mathbf{A}_{2,2} \leftarrow{ }_{R} \mathbb{Z}_{q}^{n \times m}$. For $j=1, \ldots, N$, define $\overline{\mathbf{A}}_{j}=\left(\mathbf{A}_{1} \| \mathbf{A}_{2,1}+j \mathbf{A}_{2,2}\right)$, extract a random basis $\mathbf{T}_{\overline{\mathbf{A}}_{j}} \leftarrow \operatorname{ExtRndBasis}\left(\overline{\mathbf{A}}_{j}, \mathbf{T}_{\mathbf{A}_{1}}, s\right)$. Finally, $\mathcal{B}$ gives the group public key $g p k=\left\{\mathbf{A}_{1}, \mathbf{A}_{2,1}, \mathbf{A}_{2,2}, \mathbf{B}\right\}$, and the group members' secret keys gsk $=\left\{g s k_{j}=\mathbf{T}_{\overline{\mathbf{A}}_{j}}\right\}_{j \in\{1, \ldots, N\}}$ to $\mathcal{A}$. Note that the distributions of $g p k, \mathbf{g s k}$ are statistically close to that in Game $G_{2}$ and $G_{3}$ by Proposition 4 .

When generating the challenge signature, $\mathcal{B}$ behaves the same as the challenger in $G_{2}$, except that it computes $\mathbf{c}=p \hat{\mathbf{u}}+\mathbf{x}_{1}^{*}$. We note that if $(\hat{\mathbf{B}}, \hat{\mathbf{u}})$ is a LWE tuple with respect to the error distribution $\chi_{\alpha}$, $\mathbf{c}$ is the same as in $G_{2}$. Otherwise, we have that $p \hat{\mathbf{u}}$ is uniformly distributed over $\mathbb{Z}_{q}^{m}$ (since $p, q$ are primes, and $p<q$ ), which shows that $\mathbf{c}$ has the same distribution as in $G_{3}$. If $\mathcal{A}$ can distinguish $G_{2}$ from $G_{3}$ with advantage $\epsilon$, then $\mathcal{B}$ can break the LWE assumption with advantage $\epsilon-\operatorname{negl}(k)$.

In Game $G_{4}$, the challenger behaves almost the same as in $G_{3}$, except that it randomly chooses $\mathbf{c}^{*} \leftarrow_{R} \mathbb{Z}_{q}^{m}$.

Lemma 5. In Game $G_{4}$, the probability that $b^{\prime}=b$ is exactly $1 / 2$.

Proof. The claim follows from the fact that the signature $\sigma^{*}$ in $G_{4}$ is independent from the choice of $i_{b}$.

Theorem 7. Traceability] Under the SIS assumption, our group signature $\mathcal{G S}$ is fully traceable in the random oracle model.

Proof. Assume that there is an adversary $\mathcal{A}$ that breaks the full traceability of $\mathcal{G S}$, we construct an algorithm $\mathcal{B}$ breaking the SIS assumption. Formally, $\mathcal{B}$ is given a matrix $\hat{\mathbf{A}} \in \mathbb{Z}_{q}^{m}$ and tries to find a solution $\hat{\mathbf{x}} \in \mathbb{Z}_{q}^{m}$ such that $\|\hat{\mathbf{x}}\| \leq \operatorname{poly}(m)$ and $\hat{\mathbf{A}} \hat{\mathbf{x}}=\mathbf{0}$.

Setup. $\mathcal{B}$ randomly chooses $\mathbf{R} \leftarrow_{R}\{-1,1\}^{m \times m}$ and $j^{*} \leftarrow_{R}\left\{-4 m^{2.5} N+\right.$ $\left.1, \ldots, 4 m^{2.5} N-1\right\}$, and computes $\left(\mathbf{A}_{2,2}, \mathbf{T}_{\mathbf{A}_{2,2}}\right) \leftarrow \operatorname{TrapGen}(n, m, q)$. Then, it sets $\mathbf{A}_{1}=\hat{\mathbf{A}}$ and $\mathbf{A}_{2,1}=\mathbf{A}_{1} \mathbf{R}-j^{*} \mathbf{A}_{2,2}$. Finally, compute $\left(\mathbf{B}, \mathbf{T}_{\mathbf{B}}\right) \leftarrow \operatorname{SuperSamp}\left(n, m, q, \mathbf{A}_{1}, \mathbf{0}\right)$, and give the group public key $g p k=$ $\left\{\mathbf{A}_{1}, \mathbf{A}_{2,1}, \mathbf{A}_{2,2}, \mathbf{B}\right\}$, and the group manager secret key gmsk $=\mathbf{T}_{\mathbf{B}}$ to the adversary $\mathcal{A}$. 
Secret Key Queries. Upon receiving the secret key query for user $j$ from $\mathcal{A}, \mathcal{B}$ aborts if $j=j^{*}$ or $j \notin\{1, \ldots, N\}$. Otherwise, it defines $\overline{\mathbf{A}}_{j}=$ $\left(\mathbf{A}_{1} \| \mathbf{A}_{2,1}+j^{*} \mathbf{A}_{2,2}\right)=\left(\mathbf{A}_{1} \| \mathbf{A}_{1} \mathbf{R}+\left(j-j^{*}\right) \mathbf{A}_{2,2}\right)$, extracts a random basis $\mathbf{T}_{\overline{\mathbf{A}}_{j}} \leftarrow$ ExtBasisRight $\left(\overline{\mathbf{A}}_{j}, \mathbf{R}, \mathbf{T}_{\mathbf{A}_{2,2}}, s\right),{ }^{2}$ and returns it to $\mathcal{A}$.

Sign Queries. Upon receiving a signing query for message $M$ under user $j$ from $\mathcal{A}, \mathcal{B}$ returns $\perp$ if $j \notin\{1, \ldots, N\}$. Else if $j=j^{*}, \mathcal{B}$ generates a signature on $M$ using the NIZKPoK simulators for $\pi_{1}$ and $\pi_{2}$ (i.e., by simply choosing $\mathbf{c} \leftarrow R \mathbb{Z}_{q}^{m}$ and $\left.\mathbf{x}_{2} \leftarrow_{R} D_{\mathbb{Z}^{m}, s}\right)$. Otherwise, it generates the signature by first extracting the $j$-th user's secret key as in answering the secret key queries.

Forge. Upon receiving a forged valid signature $\sigma=\left(\mathbf{c}, \mathbf{x}_{2}, \pi_{1}, \pi_{2}\right)$ with probability $\epsilon, \mathcal{B}$ extracts the knowledge $\mathbf{e}, \mathbf{x}_{1}$ and $\mathbf{v}_{j}$ with norm at most $4 \eta m^{2}$ by programming the random oracle twice to generate two different "challenges". By the forking lemma of $[12], \mathcal{B}$ can succeed with probability at least $\epsilon\left(\epsilon / q_{h}-2^{-t}\right)$, where $q_{h}$ is the maximum number of hash queries of $\mathcal{A}$. Then, $\mathcal{B}$ decrypts $\mathbf{c}$ using $\mathbf{T}_{\mathbf{B}}$, and obtains $\left(\mathbf{e}^{\prime}, \mathbf{x}_{1}^{\prime}\right)$, and distinguishes the following cases:

- If $\left(\mathbf{x}_{1}^{\prime}, \mathbf{e}^{\prime}\right) \neq\left(\mathbf{x}_{1}, \mathbf{e}\right)$, we have $\mathbf{A}_{1} \mathbf{c}=p \mathbf{A}_{1} \mathbf{e}+\mathbf{A}_{1} \mathbf{x}_{1}=p \mathbf{A}_{1} \mathbf{e}^{\prime}+\mathbf{A}_{1} \mathbf{x}_{1}^{\prime}$. Thus, $\hat{\mathbf{x}}=p\left(\mathbf{e}-\mathbf{e}^{\prime}\right)+\left(\mathbf{x}_{1}-\mathbf{x}_{1}^{\prime}\right)$ is a solution of the SIS problem, $\mathcal{B}$ returns $\hat{\mathbf{x}}$ as its own solution. Note that in this case, we have $\|\hat{\mathbf{x}}\| \leq 8(p+1) \eta m^{2}=$ $m^{8} \cdot \omega\left(\log ^{3} m\right)$.

- Otherwise, if $\left(\mathbf{x}_{1}^{\prime}, \mathbf{e}^{\prime}\right)=\left(\mathbf{x}_{1}, \mathbf{e}\right)$, we have $\mathbf{A}_{1} \mathbf{x}_{1}+\mathbf{A}_{2,1} \mathbf{x}_{2}+j \mathbf{A}_{2,2} \mathbf{x}_{2}=\mathbf{0}$ according to equation (2) of $\pi_{2}$, where $j=\sum_{i=0}^{\ell-1} v_{i} \bar{\beta}^{i}$ and $\mathbf{v}_{h}=$ $\left(v_{0}, \ldots, v_{\ell-1}\right)$. A simple calculation indicates that $|j|<4 m^{2.5} N<q$ (we note that $\left.\left\|\mathbf{v}_{h}\right\| \leq 4 \eta m^{2}=4 \beta m^{2.5}\right)$. Since $\mathbf{A}_{2,2} \mathbf{x}_{2} \neq \mathbf{0}$ and $q$ is a prime, the open algorithm will always output $j$, namely, it will never output $\perp$. In addition, if $j \neq j^{*}, \mathcal{B}$ aborts. Otherwise, $\mathcal{B}$ returns $\hat{\mathbf{x}}=\mathbf{x}_{1}+\mathbf{R} \mathbf{x}_{2}$ as its own solution.

Since $j^{*}$ is randomly chosen from $\left\{-4 m^{2.5} N+1, \ldots, 4 m^{2.5} N-1\right\}$, the probability that $j^{*}=j$ is at least $\frac{1}{8 m^{2.5} N}$. Conditioned on $j^{*}=j$, we have $\mathbf{A}_{1} \mathbf{x}_{1}+\mathbf{A}_{2,1} \mathbf{x}_{2}+j \mathbf{A}_{2,2} \mathbf{x}_{2}=\mathbf{A}_{1} \mathbf{x}_{1}+\mathbf{A}_{1} \mathbf{R} \mathbf{x}_{2}=\mathbf{0}$, which shows that $\hat{\mathbf{x}}=\mathbf{x}_{1}+\mathbf{R} \mathbf{x}_{2}$ is a solution of the SIS problem, in particular, we have $\|\hat{\mathbf{x}}\| \leq \eta m^{2.5} \cdot \omega(\sqrt{\log m})=m^{4.5} \omega\left(\log ^{2} m\right)$ by $[2$, Lem.5].

In all, the probability that $\mathcal{B}$ solves the SIS problem is at least $\frac{\epsilon\left(\epsilon / q_{h}-2^{-t}\right)}{8 m^{2.5} N}$, which is non-negligible if $\epsilon$ is non-negligible. Moreover, since the norm of $\hat{\mathbf{x}}$ is at most $m^{8} \cdot \omega\left(\log ^{3} m\right)$ and $q \geq m^{8.5} \cdot \omega\left(\log ^{2.5} m\right)$, we have that the security of our scheme is based on the hardness of the SIVP problem in the worst case to within a polynomial approximation factor, by Proposition 2 .

Acknowledgments. We would like to thank Kang Yang for his helpful comments on the related work of group signatures, and the anonymous reviewers of PKC 2015 for kind suggestions on our paper. We are also grateful to Sherman Chow for bringing their work to our attention.

\footnotetext{
${ }^{2}$ Recall that $\mathbf{T}_{\mathbf{A}_{2,2}}$ is also a short basis of $\boldsymbol{\Lambda}_{q}^{\perp}\left(j \cdot \mathbf{A}_{2,2}\right)$ for all $j \neq 0 \bmod q$.
} 


\section{References}

1. Abe, M., Fuchsbauer, G., Groth, J., Haralambiev, K., Ohkubo, M.: Structurepreserving signatures and commitments to group elements. In: Rabin, T. (ed.) CRYPTO 2010. LNCS, vol. 6223, pp. 209-236. Springer, Heidelberg (2010)

2. Agrawal, S., Boneh, D., Boyen, X.: Efficient lattice (H)IBE in the standard model. In: Gilbert, H. (ed.) EUROCRYPT 2010. LNCS, vol. 6110, pp. 553-572. Springer, Heidelberg (2010)

3. Agrawal, S., Boneh, D., Boyen, X.: Lattice basis delegation in fixed dimension and shorter-ciphertext hierarchical IBE. In: Rabin, T. (ed.) CRYPTO 2010. LNCS, vol. 6223, pp. 98-115. Springer, Heidelberg (2010)

4. Agrawal, S., Freeman, D.M., Vaikuntanathan, V.: Functional encryption for inner product predicates from learning with errors. In: Lee, D.H., Wang, X. (eds.) ASIACRYPT 2011. LNCS, vol. 7073, pp. 21-40. Springer, Heidelberg (2011)

5. Ajtai, M.: Generating hard instances of lattice problems (extended abstract). In: 28th Annual ACM Symposium on Theory of Computing (STOC), pp. 99-108. ACM, New York (1996)

6. Ajtai, M.: Generating hard instances of the short basis problem. In: Wiedermann, J., Van Emde Boas, P., Nielsen, M. (eds.) ICALP 1999. LNCS, vol. 1644, pp. 1-9. Springer, Heidelberg (1999)

7. Alwen, J., Peikert, C.: Generating shorter bases for hard random lattices. In: STACS, pp. 75-86 (2009)

8. Applebaum, B., Cash, D., Peikert, C., Sahai, A.: Fast cryptographic primitives and circular-secure encryption based on hard learning problems. In: Halevi, S. (ed.) CRYPTO 2009. LNCS, vol. 5677, pp. 595-618. Springer, Heidelberg (2009)

9. Ateniese, G., Camenisch, J.L., Joye, M., Tsudik, G.: A practical and provably secure coalition-resistant group signature scheme. In: Bellare, M. (ed.) CRYPTO 2000. LNCS, vol. 1880, pp. 255-270. Springer, Heidelberg (2000)

10. Ateniese, G., Song, D., Tsudik, G.: Quasi-efficient revocation of group signatures. In: Blaze, M. (ed.) FC 2002. LNCS, vol. 2357, pp. 183-197. Springer, Heidelberg (2002)

11. Bellare, M., Micciancio, D., Warinschi, B.: Foundations of group signatures: formal definitions, simplified requirements, and a construction based on general assumptions. In: Biham, E. (ed.) EUROCRYPT 2003. LNCS, vol. 2656, pp. 614-629. Springer, Heidelberg (2003)

12. Bellare, M., Neven, G.: Multi-signatures in the plain public-key model and a general forking lemma. In: 13th ACM Conference on Computer and Communications Security (CCS), pp. 390-399. ACM, New York (2006)

13. Bellare, M., Rogaway, P.: Random oracles are practical: a paradigm for designing efficient protocols. In: 1st ACM Conference on Computer and Communications Security (CCS), pp. 62-73. ACM Press (1993)

14. Benhamouda, F., Camenisch, J., Krenn, S., Lyubashevsky, V., Neven, G.: Better zero-knowledge proofs for lattice encryption and their application to group signatures. In: Sarkar, P., Iwata, T. (eds.) ASIACRYPT 2014. LNCS, vol. 8873, pp. 551-572. Springer, Heidelberg (2014)

15. Boneh, D., Boyen, X.: Short signatures without random oracles. In: Cachin, C., Camenisch, J.L. (eds.) EUROCRYPT 2004. LNCS, vol. 3027, pp. 56-73. Springer, Heidelberg (2004)

16. Boneh, D., Boyen, X., Shacham, H.: Short group signatures. In: Franklin, M. (ed.) CRYPTO 2004. LNCS, vol. 3152, pp. 41-55. Springer, Heidelberg (2004) 
17. Boneh, D., Nikolaenko, V., Segev, G.: Attribute-based encryption for arithmetic circuits. Cryptology ePrint Archive, Report 2013/669 (2013)

18. Boneh, D., Shacham, H.: Group signatures with verifier-local revocation. In: 11th ACM Conference on Computer and Communications Security (CCS), pp. 168-177. ACM, New York (2004)

19. Boyen, X.: Lattice mixing and vanishing trapdoors: a framework for fully secure short signatures and more. In: Nguyen, P.Q., Pointcheval, D. (eds.) PKC 2010. LNCS, vol. 6056, pp. 499-517. Springer, Heidelberg (2010)

20. Boyen, X., Waters, B.: Compact group signatures without random oracles. In: Vaudenay, S. (ed.) EUROCRYPT 2006. LNCS, vol. 4004, pp. 427-444. Springer, Heidelberg (2006)

21. Boyen, X., Waters, B.: Full-domain subgroup hiding and constant-size group signatures. In: Okamoto, T., Wang, X. (eds.) PKC 2007. LNCS, vol. 4450, pp. 1-15. Springer, Heidelberg (2007)

22. Brakerski, Z., Gentry, C., Vaikuntanathan, V.: Fully homomorphic encryption without bootstrapping. In: Innovations in Theoretical Computer Science, ITCS, pp. 309-325 (2012)

23. Brakerski, Z., Vaikuntanathank, V. : Efficient fully homomorphic encryption from (standard) LWE. In: IEEE 52nd Annual Symposium on Foundations of Computer Science (FOCS), pp. 97-106 (2011)

24. Brakerski, Z., Vaikuntanathan, V.: Lattice-based FHE as secure as PKE. In: 5th Conference on Innovations in Theoretical Computer Science (ITCS), pp. 1-12. ACM, New York (2014)

25. Bresson, E., Stern, J.: Efficient revocation in group signatures. In: Kim, K. (ed.) PKC 2001. LNCS, vol. 1992, pp. 190-206. Springer, Heidelberg (2001)

26. Brickell, E., Camenisch, J., Chen, L.: Direct anonymous attestation. In: 11th ACM Conference on Computer and Communications Security (CCS), pp. 132-145. ACM Press (2004)

27. Camenisch, J.L., Lysyanskaya, A.: Dynamic accumulators and application to efficient revocation of anonymous credentials. In: Yung, M. (ed.) CRYPTO 2002. LNCS, vol. 2442, pp. 61-76. Springer, Heidelberg (2002)

28. Camenisch, J.L., Lysyanskaya, A.: Signature schemes and anonymous credentials from bilinear maps. In: Franklin, M. (ed.) CRYPTO 2004. LNCS, vol. 3152, pp. 56-72. Springer, Heidelberg (2004)

29. Camenisch, J., Neven, G., Rückert, M.: Fully anonymous attribute tokens from lattices. In: Visconti, I., De Prisco, R. (eds.) SCN 2012. LNCS, vol. 7485, pp. 57-75. Springer, Heidelberg (2012)

30. Canetti, R., Halevi, S., Katz, J.: Chosen-ciphertext security from identity-based encryption. In: Cachin, C., Camenisch, J.L. (eds.) EUROCRYPT 2004. LNCS, vol. 3027, pp. 207-222. Springer, Heidelberg (2004)

31. Cash, D., Hofheinz, D., Kiltz, E., Peikert, C.: Bonsai trees, or how to delegate a lattice basis. In: Gilbert, H. (ed.) EUROCRYPT 2010. LNCS, vol. 6110, pp. 523-552. Springer, Heidelberg (2010)

32. Chaum, D., van Heyst, E.: Group signatures. In: Davies, D.W. (ed.) EUROCRYPT 1991. LNCS, vol. 547, pp. 257-265. Springer, Heidelberg (1991)

33. Chen, L., Li, J.: Flexible and scalable digital signatures in TPM 2.0. In: 20th ACM Conference on Computer and Communications Security (CCS), pp. 37-48. ACM Press (2013)

34. Fiat, A., Shamir, A.: How to prove yourself: practical solutions to identification and signature problems. In: Odlyzko, A.M. (ed.) CRYPTO 1986. LNCS, vol. 263, pp. 186-194. Springer, Heidelberg (1987) 
35. Gentry, C., Gorbunov, S., Halevi, S., Vaikuntanathan, V., Vinayagamurthy, D.: How to compress (reusable) garbled circuits. Cryptology ePrint Archive, Report 2013/687 (2013)

36. Gentry, C., Peikert, C., Vaikuntanathan, V.: Trapdoors for hard lattices and new cryptographic constructions. In: 40th Annual ACM Symposium on Theory of Computing (STOC), pp. 197-206. ACM, New York (2008)

37. Gordon, S.D., Katz, J., Vaikuntanathan, V.: A group signature scheme from lattice assumptions. In: Abe, M. (ed.) ASIACRYPT 2010. LNCS, vol. 6477, pp. 395-412. Springer, Heidelberg (2010)

38. Groth, J.: Simulation-sound NIZK proofs for a practical language and constant size group signatures. In: Lai, X., Chen, K. (eds.) ASIACRYPT 2006. LNCS, vol. 4284, pp. 444-459. Springer, Heidelberg (2006)

39. Groth, J.: Fully anonymous group signatures without random oracles. In: Kurosawa, K. (ed.) ASIACRYPT 2007. LNCS, vol. 4833, pp. 164-180. Springer, Heidelberg (2007)

40. T.C. Group. TCG TPM specification 1.2. (2003). http://www.trustedcomputinggroup.org

41. T.C. Group. TCG TPM specification 2.0. (2013). http://www.trustedcomputinggroup.org/resources/tpm_library_specification

42. I.P.W. Group, VSC Project. Dedicated short range communications (DSRC) (2003)

43. Laguillaumie, F., Langlois, A., Libert, B., Stehlé, D.: Lattice-based group signatures with logarithmic signature size. In: Sako, K., Sarkar, P. (eds.) ASIACRYPT 2013, Part II. LNCS, vol. 8270, pp. 41-61. Springer, Heidelberg (2013)

44. Langlois, A., Ling, S., Nguyen, K., Wang, H.: Lattice-based group signature scheme with verifier-local revocation. In: Krawczyk, H. (ed.) PKC 2014. LNCS, vol. 8383, pp. 345-361. Springer, Heidelberg (2014)

45. Libert, B., Peters, T., Yung, M.: Group signatures with almost-for-free revocation. In: Safavi-Naini, R., Canetti, R. (eds.) CRYPTO 2012. LNCS, vol. 7417, pp. 571589. Springer, Heidelberg (2012)

46. Libert, B., Peters, T., Yung, M.: Scalable group signatures with revocation. In: Pointcheval, D., Johansson, T. (eds.) EUROCRYPT 2012. LNCS, vol. 7237, pp. 609-627. Springer, Heidelberg (2012)

47. Ling, S., Nguyen, K., Stehlé, D., Wang, H.: Improved zero-knowledge proofs of knowledge for the ISIS problem, and applications. In: Kurosawa, K., Hanaoka, G. (eds.) PKC 2013. LNCS, vol. 7778, pp. 107-124. Springer, Heidelberg (2013)

48. Ling, S., Nguyen, K., Wang, H.: Group signatures from lattices: simpler, tighter, shorter, ring-based. In: Katz, J. (ed.) PKC 2015. LNCS, vol. 9020, pp. xx-yy. Springer, Heidelberg (2015)

49. Lyubashevsky, V.: Lattice-based identification schemes secure under active attacks. In: Cramer, R. (ed.) PKC 2008. LNCS, vol. 4939, pp. 162-179. Springer, Heidelberg (2008)

50. Lyubashevsky, V.: Lattice signatures without trapdoors. In: Pointcheval, D., Johansson, T. (eds.) EUROCRYPT 2012. LNCS, vol. 7237, pp. 738-755. Springer, Heidelberg (2012)

51. Micciancio, D., Mol, P.: Pseudorandom knapsacks and the sample complexity of LWE search-to-decision reductions. In: Rogaway, P. (ed.) CRYPTO 2011. LNCS, vol. 6841, pp. 465-484. Springer, Heidelberg (2011)

52. Micciancio, D., Peikert, C.: Trapdoors for lattices: simpler, tighter, faster, smaller. In: Pointcheval, D., Johansson, T. (eds.) EUROCRYPT 2012. LNCS, vol. 7237, pp. 700-718. Springer, Heidelberg (2012) 
53. Micciancio, D., Regev, O.: Worst-case to average-case reductions based on gaussian measures. SIAM J. Comput. 37, 267-302 (2007)

54. Micciancio, D., Vadhan, S.P.: Statistical zero-knowledge proofs with efficient provers: lattice problems and more. In: Boneh, D. (ed.) CRYPTO 2003. LNCS, vol. 2729, pp. 282-298. Springer, Heidelberg (2003)

55. Peikert, C.: Public-key cryptosystems from the worst-case shortest vector problem: extended abstract. In: 41st Annual ACM Symposium on Theory of Computing (STOC), pp. 333-342. ACM, New York (2009)

56. Peikert, C., Rosen, A.: Efficient collision-resistant hashing from worst-case assumptions on cyclic lattices. In: Halevi, S., Rabin, T. (eds.) TCC 2006. LNCS, vol. 3876, pp. 145-166. Springer, Heidelberg (2006)

57. Regev, O.: On lattices, learning with errors, random linear codes, and cryptography. In: 37 Annual ACM Symposium on Theory of Computing (STOC), pp. 84-93. ACM, New York (2005)

58. Stern, J.: A new paradigm for public key identification. IEEE Transactions on Information Theory 42(6), 1757-1768 (1996) 